\title{
Nanomolar, noncovalent antagonism of hedgehog cholesterolysis: exception to the "irreversibility rule" for protein autoprocessing inhibition.
}

Andrew G. Wagner, ${ }^{1}$ Robert T. Stagnitta, ${ }^{1}$ Zihan Xu, ${ }^{1}$ John L. Pezzullo, ${ }^{2}$ Nabin Kandel, ${ }^{3}$ José-Luis Giner, ${ }^{2}$ Douglas F. Covey, ${ }^{4}$ Chunyu Wang, ${ }^{3}$ Brian P. Callahan ${ }^{1^{*}}$

(1) Department of Chemistry, Binghamton University, State University of New York, Binghamton, New York 13902, United States

(2) Department of Chemistry, State University of New York College of Environmental Science and Forestry, Syracuse, New York

13210, United States (3) Department of Biological Sciences, Center for Biotechnology and Interdisciplinary Studies, Rensselaer Polytechnic Institute, 110 8th Street, Troy, New York 12180, United States (4) Department of Developmental Biology, Taylor Family Institute for Innovative Psychiatric Research, 660 South Euclid Avenue, St. Louis, Missouri 63110, United States

*Corresponding Author: Brian P. Callahan - Department of Chemistry, Binghamton University, State University of New York, Binghamton, New York 13902, United States; callahan@binghamton.edu

$\begin{array}{lll}\text { Figure } \mathrm{S} 1 & \text { Inhibition } \mathrm{C}-\mathrm{H}-\mathrm{Y} \text { cholesterolysis by 1-4 and } \mathbf{8} & \mathrm{S} 2\end{array}$

Figure $\mathrm{S2} \quad t \mathrm{SP}$ is a substrate analog and photoaffinity probe for HhC S3

Figure S3 Inhibitor 1 increases FRET of C-H-Y, but not C-HINT-Y, C-SRR-Y, or C-Y S4

Figure $S 4$ HhC point mutants show a wide range of binding affinity for $t B T-H B T$ S5

Figure S5 Inhibitor 1 competitively slows the rate of paracatalytic induction of $\mathrm{C}-\mathrm{H}-\mathrm{Y}$ by HAC8 $\quad \mathrm{S6}$

Figure 56 SDS-PAGE analysis of purified FRET proteins used in this study $\quad$ S7

Figure S7 Dose response inhibition of $\mathrm{C}-\mathrm{H}-\mathrm{Y}$ cholesterolysis by 1-12 $\quad$ S8

A. Chemicals and solvents $\quad$ S9

B. Chemical library $\quad$ S10

C. Generation of FRET-reporter expression plasmids $\quad$ S10

D. Expression and purification of FRET-active HhC reporter constructs $\quad$ S10

E. Cholesterolysis activity and inhibition assays with $\mathrm{C}-\mathrm{H}-\mathrm{Y} \quad \mathrm{S} 10$

F. Steady state kinetics of cholesterolysis $\quad$ S11

G. Inhibitor $\mathrm{IC}_{50}$ determination $\quad$ S11

$\mathrm{H}$. Inhibitor binding affinity determined by $\triangle \mathrm{FRET}$ assay $\quad$ S11

I. Photoaffinity labeling of product HhC by $t S P$

J. Generation of D.me HhC homology model $\quad$ S13

K. Synthesis of inhibitor analogs $\quad$ S13-15

L. ${ }^{1} \mathrm{H},{ }^{13} \mathrm{C}$ NMR spectra for compounds 5-12

M. LCMS data for compounds 5-12

References $\quad$ S33 


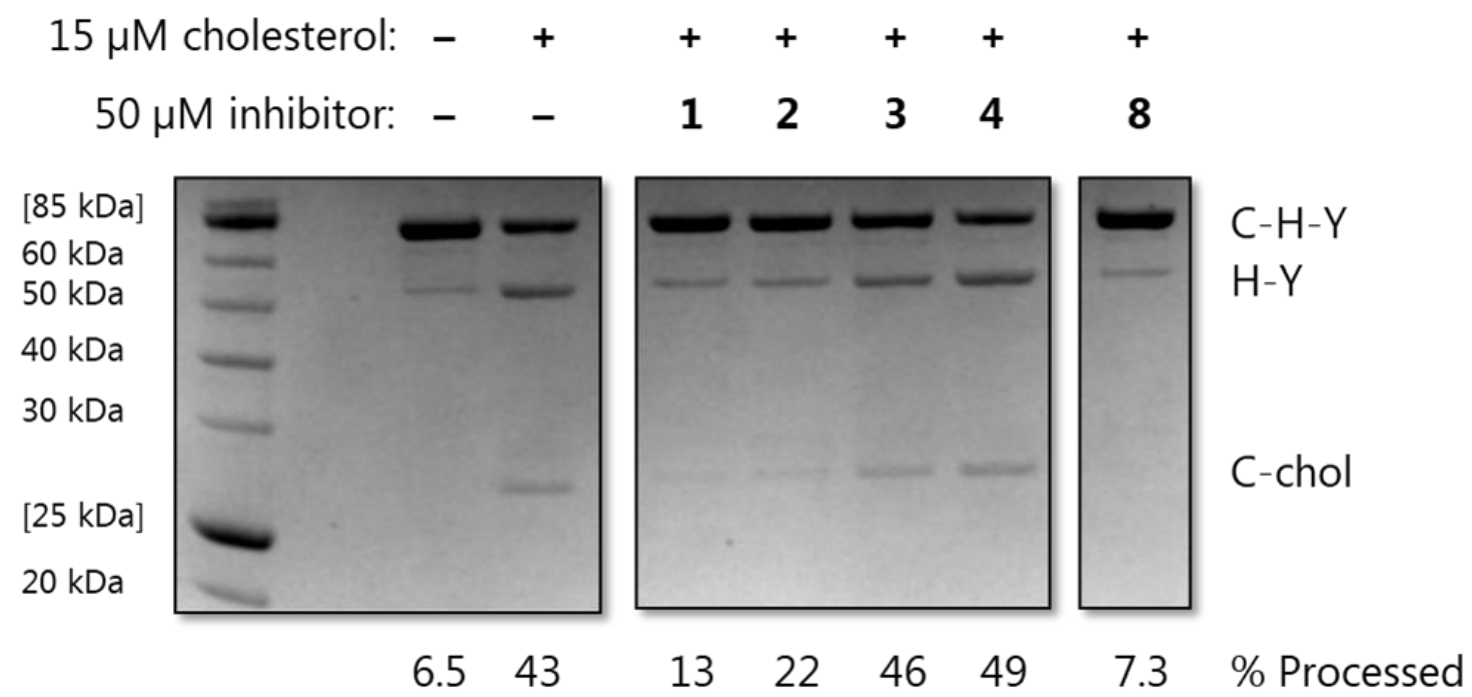

Figure S1: Inhibition of $\mathrm{C}-\mathrm{H}-\mathrm{Y}$ cholesterolysis by 1-4 and 8. Reactions were incubated for 20 minutes at $30{ }^{\circ} \mathrm{C}$ and contained $0.5 \mu \mathrm{M} \mathrm{C}-\mathrm{H}-\mathrm{Y}+/$ - cholesterol and inhibitor as indicated. Densitometry of precursor and product bands were used to calculate the extent of reaction. 

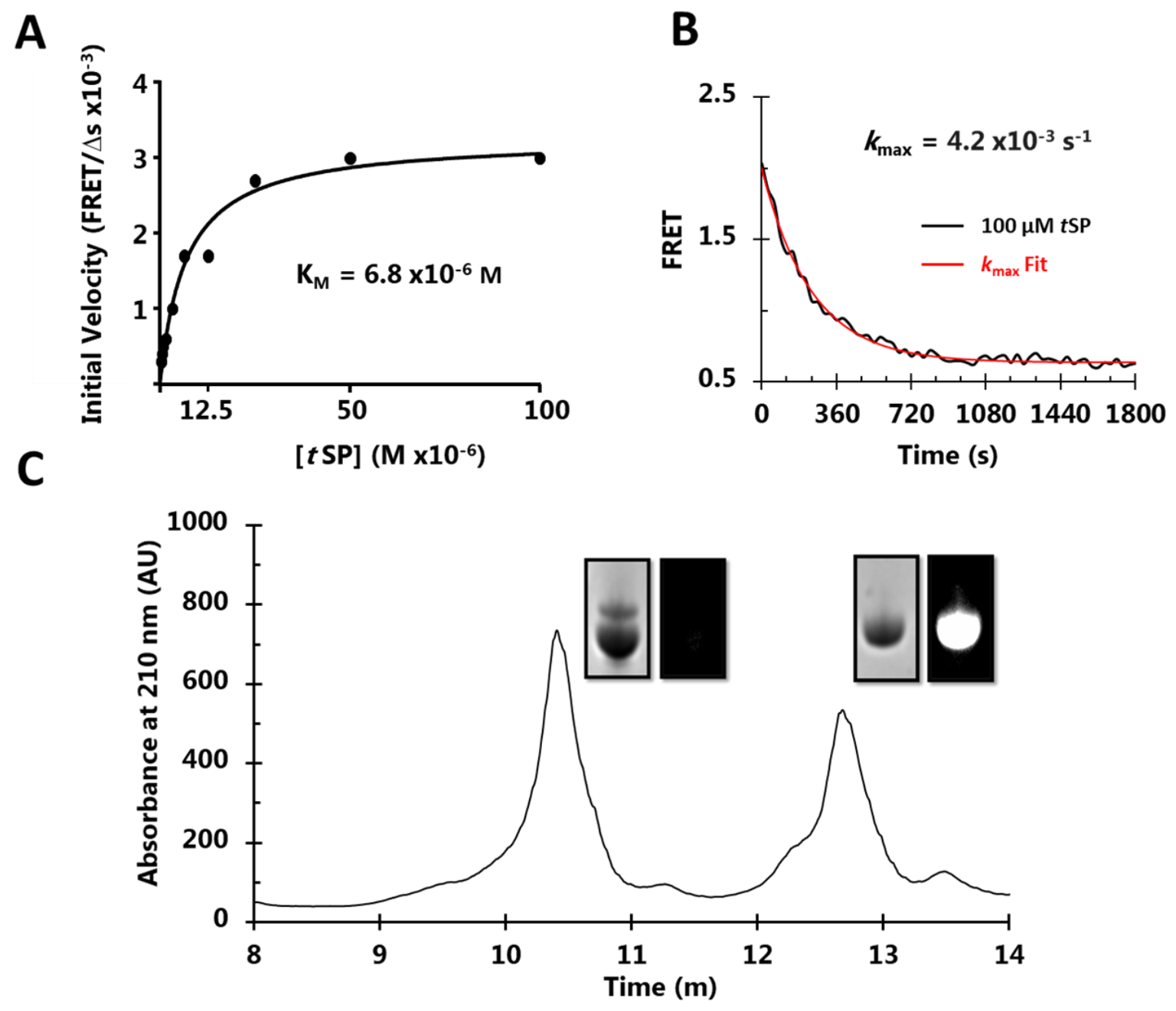

Figure S2: $t S P$ is a substrate analog and photoaffinity probe for HhC. (A) Plot of initial reaction velocity of $\mathrm{C}-\mathrm{H}-\mathrm{Y}$ as a function of increasing $t \mathrm{SP}$ substrate concentration. (B) First order kinetics of $\mathrm{C}-\mathrm{H}-\mathrm{Y}$ sterolysis with saturating $t \mathrm{SP}(100 \mu \mathrm{M})$ to determine $k_{\max }$, the maximum rate of sterolysis. (C) Separation of HhC and HhC: $t S P$ adduct by reverse phase HPLC. The HhC:tSP adduct elutes at 12.7 minutes, two minutes after unlabeled HhC. (Inset) Confirmation of HhC:tSP adduct by click labeling of the side-chain alkyne of $t$ SP. HPLC fractions were combined with an azide-modified fluorophore (ClickChemistryTools OG-488 azide \#1264-1) under copper catalyzed click chemistry conditions, separated by SDS-PAGE, and visualized with Coomassie staining and UV imaging. 


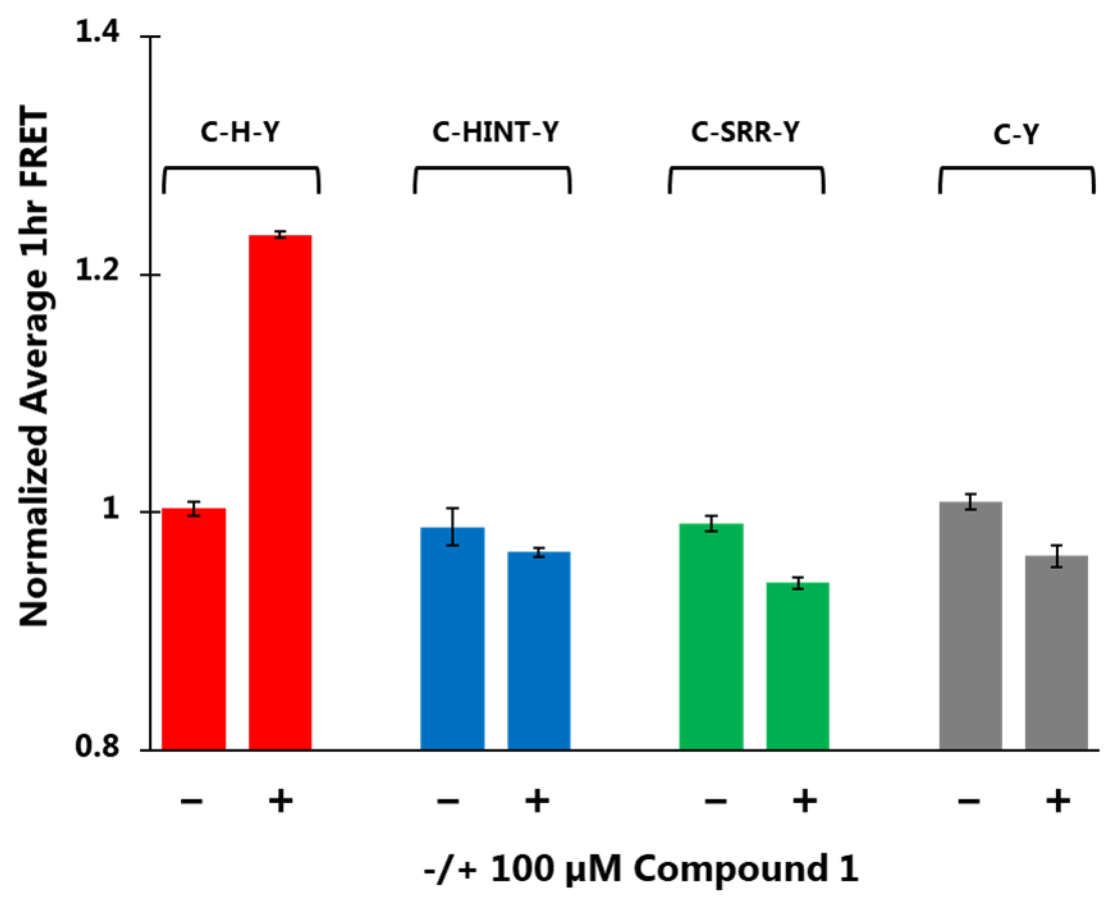

Figure S3: Inhibitor 1 increases FRET of C-H-Y, but not C-HINT-Y, C-SRR-Y, or C-Y. Average FRET change $(n=6)$ for the indicated reporter constructs $+/$ - inhibitor $\mathbf{1}(100 \mu \mathrm{M})$. C-HINT-Y and C-SRR-Y are truncation mutants of $\mathrm{HhC}$; the $\mathrm{C}-\mathrm{Y}$ construct includes CFP and YFP only and is used here as a negative control. 


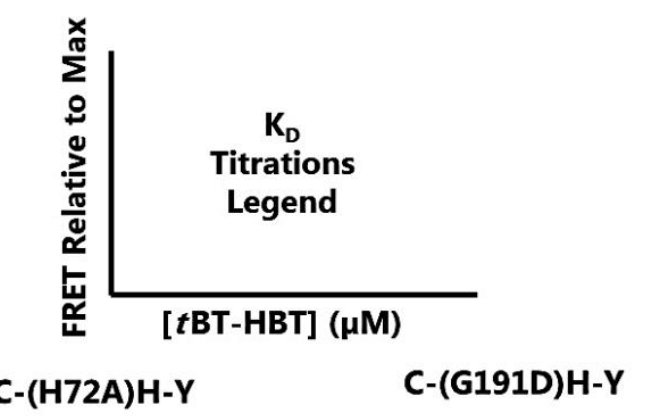

C-H-Y

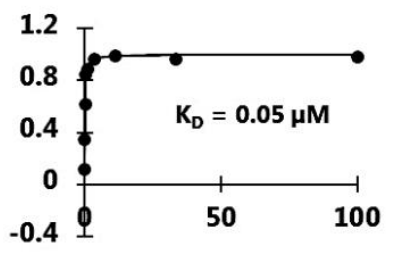

C-(Y195)H-Y

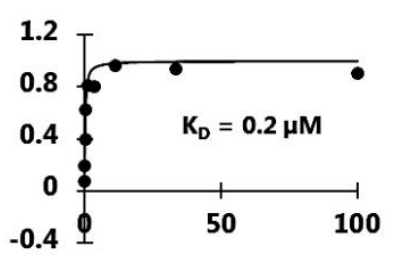

C-(H193Y)H-Y

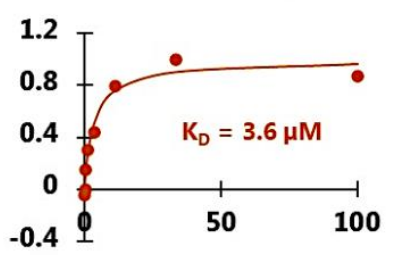

C-(C1A)H-Y

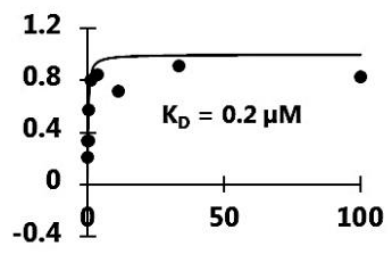

C-(L128A)H-Y

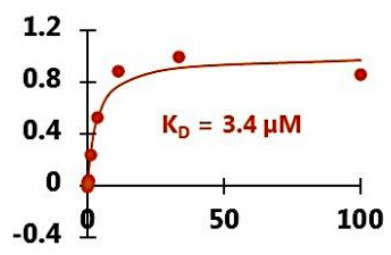

C-(W194A)H-Y

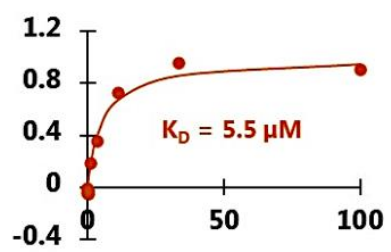

Figure S4: HhC point mutants show a wide range of binding affinity for $t$ BT-HBT. $\triangle$ FRET for wild-type HhC $(\mathrm{C}-\mathrm{H}-\mathrm{Y})$ and $\mathrm{HhC}$ point mutants is plotted as a function of increasing $t \mathrm{BT}-\mathrm{HBT}$ concentration. Binding isotherms (solid line) represent the behavior expected for the $\mathrm{K}_{\mathrm{D}}$ values indicated in each graph. Mutants $\mathrm{C}-(\mathrm{L73A}) \mathrm{H}-\mathrm{Y}, \mathrm{C}-(\mathrm{F} 86 \mathrm{~A}) \mathrm{H}-\mathrm{Y}, \mathrm{C}-(\mathrm{C} 143 \mathrm{D}) \mathrm{H}-\mathrm{Y}$, and $\mathrm{C}-(\mathrm{Y} 144 \mathrm{~F}) \mathrm{H}-\mathrm{Y}$, not shown, lacked interpretable doseresponse behavior with added $t \mathrm{BT}-\mathrm{HBT}$. 


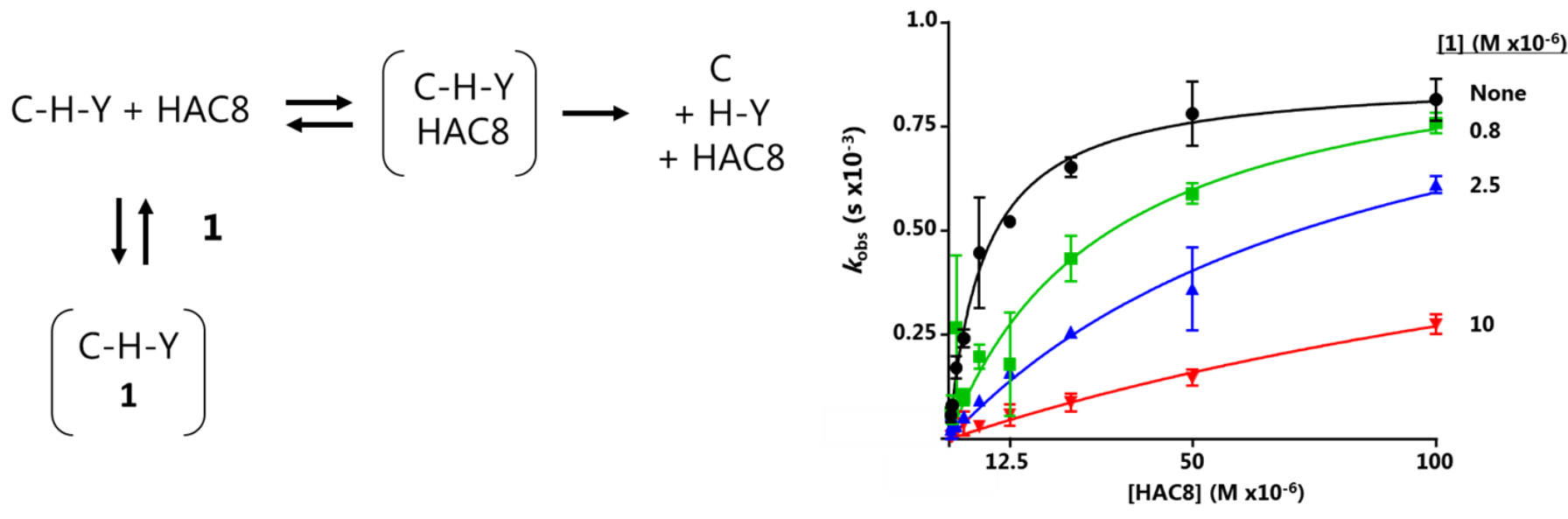

Figure S5: Inhibitor 1 competitively slows the rate of paracatalytic induction of $\mathrm{C}-\mathrm{H}-\mathrm{Y}$ autoproteolysis by HAC8. (Left) $\mathrm{C}-\mathrm{H}-\mathrm{Y}$ reaction schematic representing competitive binding of HAC8 and inhibitor 1. (Right) Plot of the first order rate constants for $\mathrm{C}-\mathrm{H}-\mathrm{Y}$ autoproteolysis (cholesterol independent) plotted as a function of increasing HAC8 concentration. Color code: $0.8 \mu \mathrm{M}, 1$ (green), $2.5 \mu \mathrm{M}, 1$ (blue) or $10 \mu \mathrm{M}, 1$ (red); absence of $\mathbf{1}$ (black). 


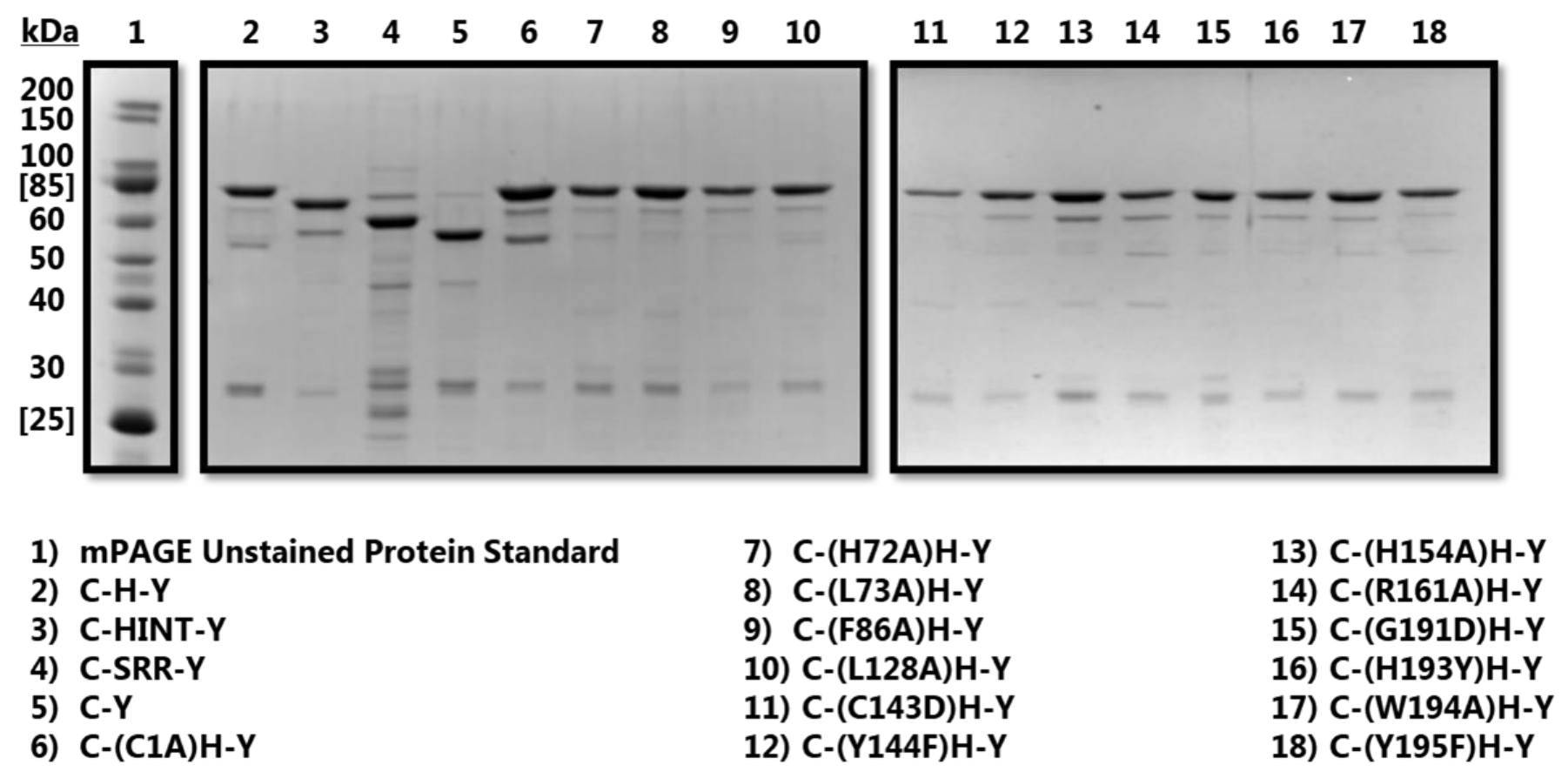

Figure S6: Ni-NTA purified FRET reporter proteins used in this study. Proteins were separated by SDSPAGE (12\%). 


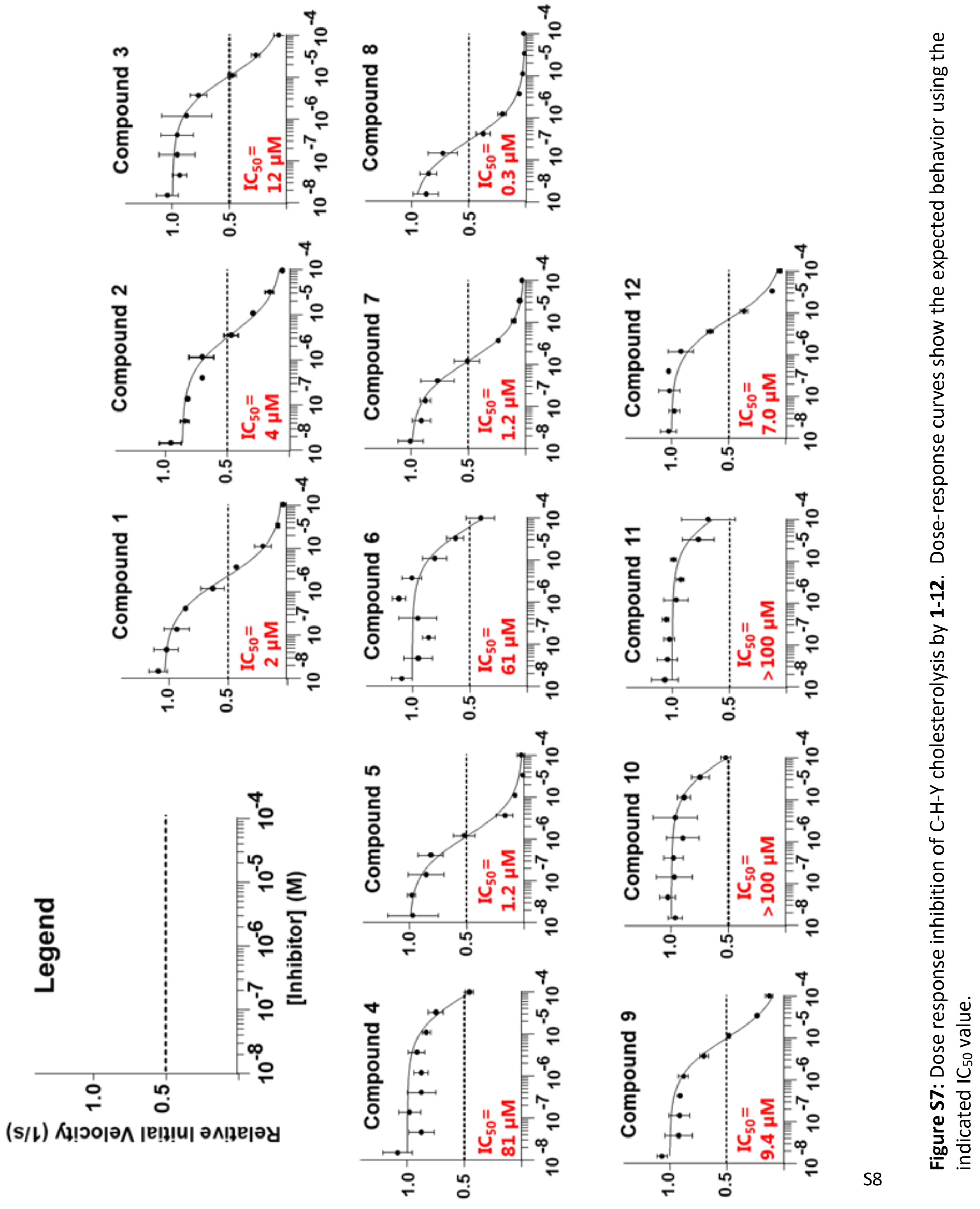




\section{Materials and Methods}

\section{Chemicals and Solvents:}

Acros Organics: Silica gel, imidazole

Alfa Aesar: Homopiperazine, 2-chlorobenzothiazole, 4-(dimethylamino)pyridine (DMAP), Lascorbic acid, 5-bromo-2-thiophenecarboxylic acid

Anatrace: n-Dodecylphosphocholine (Fos-12)

BLD Pharm: Sodium thiophene-2-carboxylate

Cambridge Isotopes: D-chloroform, d-methanol

ChemBridge, Hit2Lead: Inhibitor compounds 1-4, 1-tert-butyl-1H-pyrrole-3-carboxylic acid, Click Chemistry tools: OG 488 azide fluorophore, tris(benzyltriazolylmethyl)amine (THPTA) Corning: 96 well black polystyrene microplate（\#3650）

Cytiva: HisSpinTrap Ni 9epharose high performance spin columns

Enamine: 2-tert-butyl-1,3-oxazole-5-carboxylic acid, 5-cyanothiophene-2-carboxylic acid, 5phenylthiophene-2-carboxylic acid

Fischer Scientific: Dichloromethane, ethyl acetate, glycerol, tris(2-carboxyethyl)phosphine (TCEP), tris base

\section{G Bioscience: Dimethylformamide (DMF)}

GE Healthcare: Tetramethylethylenediamine (TEMED)

InVitria: Lysozyme

Invitrogen - 40\% acrylamide (29:1 mono:bis)

JT Baker: Methanol, sodium sulfate, copper sulfate

MP Biomedicals: Iodoacetamide

Millipore Sigma: Triton X-100

New England Biolabs: All restriction enzymes and buffers, DNA ligase and buffers, and all competent cells, plasmid miniprep kits

Oakwood Chemical: 5-tert-butylthiophene-2-carboxylic acid

Qiagen: Filtered Ni-NTA spin columns

Sigma-Aldrich: 1-ethyl-3-(3-dimethylaminopropyl) carbodiimide hydrochloride (EDC), 1,4dithiothreitol (DTT), $\beta$-mercaptoethanol (BME), L-arabinose, $1 \mathrm{H}$-pyrrole-3-carboxylic acid, Thermo Fisher Scientific: All antibiotics, isopropyl $\beta$-D-1- thiogalactopyranoside (IPTG), LB broth miller (Granulated), dimethyl sulfoxide (DMSO), LB agar (miller), Coomassie brilliant blue G-250, ammonium persulfate,

VWR: Acetic acid glacial 
B. Chemical library: Compounds used in the inhibitor screen were purchased from ChemBridge and stored at $-80{ }^{\circ} \mathrm{C}$ as $10 \mathrm{mM}$ stocks dissolved in DMSO.

C. Generation of FRET-reporter expression plasmids: The arabinose inducible pBAD33 expression plasmids $\mathrm{C}-\mathrm{H}-\mathrm{Y}, \mathrm{C}-\mathrm{Y}, \mathrm{C}-(\mathrm{C} 1 \mathrm{~A}) \mathrm{H}-\mathrm{Y}$, and $\mathrm{C}-(\mathrm{C} 143 \mathrm{D}) \mathrm{H}-\mathrm{Y}$ FRET constructs have been described. ${ }^{1-3}$ In a similar way, we prepared truncation mutants of HhC, C-HINT-Y, C-SRR-Y, and HhC point mutants, C-(H72A)H-Y, C-(Y144F)H-Y, C-(H154A)H-Y, C-(R161A)H-Y, C-(G191D)H-Y, C$(\mathrm{H} 193 \mathrm{Y}) \mathrm{H}-\mathrm{Y}$, and $\mathrm{C}-(\mathrm{Y} 195 \mathrm{~F}) \mathrm{H}-\mathrm{Y}$. Synthetic genes encoding the respective HhC mutant proteins were cloned into the pBAD33 $\mathrm{C}-\mathrm{H}-\mathrm{Y}$ expression vector as Sall/Pstl fragments, using the Xhol and Pstl sites located at $3^{\prime}$ of CFP and $5^{\prime}$ of YFP in pBAD33 C-H-Y. ${ }^{4}$ After silencing an internal Xhol site in HhC, we prepared C-(L73A)H-Y, C-(F86A)H-Y, C-(L128A)H-Y, and C-(W194A)H-Y by cloning the corresponding $\mathrm{HhC}$ mutant genes as Xhol/Pstl fragments into Xhol/Pstl digested pBAD33 C-H-Y.

D. Expression and purification of FRET-active HhC reporter constructs: The following Ni-NTA purification buffers were used in this study:

- Bacterial Cell Lysis buffer: $0.5 \%$ triton X-100, $0.05 \mathrm{M} \mathrm{K}_{2} \mathrm{HPO}_{4}, 0.4 \mathrm{M} \mathrm{NaCl}, 0.1 \mathrm{M} \mathrm{KCl}, 10$ $\%$ glycerol, $0.01 \mathrm{M}$ imidazole, $\mathrm{pH}=7.3$.

- Ni-NTA Wash buffer: $1 \mathrm{M} \mathrm{NaCl}, 0.04 \mathrm{M} \mathrm{Na}_{2} \mathrm{HPO}_{4}, 0.075 \mathrm{M}$ imidazole, $20 \%$ glycerol, $\mathrm{pH}=7.5$.

- Ni-NTA Elution buffer: $0.02 \mathrm{M} \mathrm{Na}_{2} \mathrm{HPO}_{4}, 0.5 \mathrm{M} \mathrm{NaCl}, 0.5 \mathrm{M}$ imidazole, $10 \%$ glycerol, $\mathrm{pH}=7.3$.

FRET-active HhC reporter proteins were expressed and purified from LMG-194 E. coli. ${ }^{1}$ Bacteria were seeded from a single colony into $50 \mathrm{ml}$ of LB media with chloramphenicol $(100 \mu \mathrm{g} / \mathrm{ml})$, cultured at $37^{\circ} \mathrm{C}(230 \mathrm{rpm})$ until an $\mathrm{OD}_{600}$ of 0.5 was reached. Expression was induced by adding L-arabinose $(100 \mathrm{mg})$ and the temperature was reduced to $16{ }^{\circ} \mathrm{C}$. After overnight incubation, bacteria were harvested by centrifugation $(10,000 \mathrm{xg}, 5 \mathrm{~min}$.). Cell pellets were resuspended in 3 $\mathrm{ml}$ of ice-cold Bacterial Cell Lysis buffer with $1 \mathrm{mg} / \mathrm{ml}$ lysozyme and subjected to sonication. Soluble protein was separated from insoluble debris by centrifugation (12,000 xg, $30 \mathrm{~min}$.). FRET reporter proteins, which all contain a C-terminal His 6 sequence, were purified using Cytiva $\mathrm{Ni}$ NTA spin columns. Typical volumes for each step in the purification are as follows: Ni-NTA Wash buffer, $0.5 \mathrm{ml}$; Ni-NTA Elution buffer $0.3 \mathrm{ml}$. Eluted protein was analyzed by $12 \%$ acrylamide SDSPAGE (Figure S6) with the concentration of FRET construct estimated by gel densitometry using ImageJ software.

E. Cholesterolysis activity and inhibition assays with C-H-Y: The FRET reporter system allows for continuous monitoring of cholesterolysis kinetics in multi-well plates. ${ }^{1,}{ }^{2}$ Assays (100 $\left.\mu \mathrm{l}\right)$ were carried out at $30^{\circ} \mathrm{C}$ and included $\mathrm{C}-\mathrm{H}-\mathrm{Y}\left(1 \times 10^{-7} \mathrm{M}\right)$ in cholesterolysis buffer: bis-tris $(0.02 \mathrm{M}, \mathrm{pH}$ 7.1) with ethylenediaminetetraacetic acid (EDTA, $0.005 \mathrm{M}), \quad \mathrm{NaCl}(0.1 \mathrm{M})$, $\mathrm{n}$ dodecylphosphocholine (Fos-12, $0.0015 \mathrm{M}$ ) and DTT (0.001 M). When testing potential inhibitors, $\mathrm{C}-\mathrm{H}-\mathrm{Y}$ and the compound were pre-incubated for $30 \mathrm{~min}$, then the reaction was initiated by addition of cholesterol to $1.5 \mu \mathrm{M}$ (4\% ethanol v/v). FRET activity was monitored at 120 second 
intervals. Cholesterolysis was also assayed by SDS-PAGE, following the disappearance of the 80 kDA C-H-Y precursor and appearance of two products, cholesterylated CFP (23 kDa) and HhC-YFP (56 kDa). A Bio-Rad Gel Doc EZ system was used to record gel images after UV imaging and Coomassie staining (Figure S1).

F. Steady state kinetics of cholesterolysis. $\mathrm{K}_{\mathrm{M}}$ and $k_{\max }$ values for sterolysis activity of $\mathrm{C}-\mathrm{H}-\mathrm{Y}$ were calculated separately and are based on the following kinetic scheme:

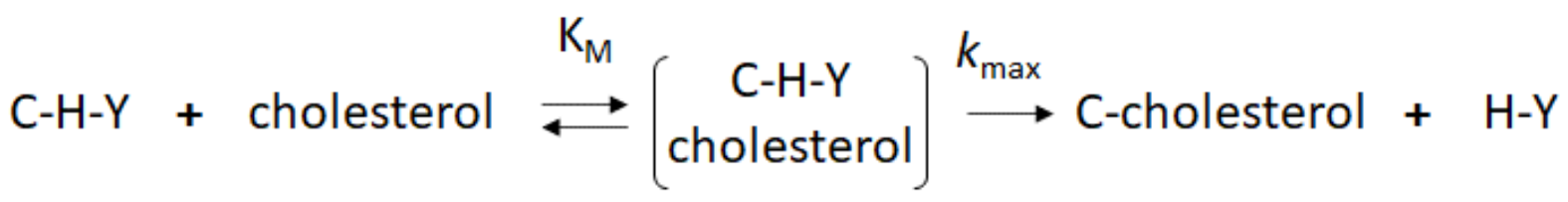

$\mathrm{K}_{\mathrm{M}}$ was derived from Michaelis-Menten type graphs of the initial rate of FRET loss $\left(\mathrm{V}_{\mathrm{o}}\right)$ plotted as a function of increasing sterol concentration (see for example, Figure S2A). Using the equation $\mathrm{V}_{\mathrm{o}}=\mathrm{V}_{\text {max }}$ [sterol $] /\left([\right.$ sterol $\left.]+\mathrm{K}_{\mathrm{M}}\right)$ we derived apparent $\mathrm{K}_{\mathrm{M}}$ values. Residual errors between the observed and calculated values of $V_{\max }$ and $\mathrm{K}_{M}$ were minimized in Excel using the Solver function. Values for the first order rate of cholesterolysis, $k_{\max }$, were determined by fitting a full kinetic trace from reactions with saturating sterol to the equation, $F R E T=A * e^{-k m a x * t}+C$ (see for example, Figure S2B).

The Michaelis-Menten steady state formalism is applied to the kinetics of cholesterolysis despite differences between the action of $\mathrm{HhC}$ autoprocessing and that of a typical multi-turnover enzyme. Autoprocessing proteins like $\mathrm{HhC}$ are attached to their polypeptide substrate and are considered single turnover catalysts. The concentration of the $\mathrm{C}-\mathrm{H}-\mathrm{Y}$ - cholesterol complex is not constant but declines with each "turnover". By using the initial rates of cholesterolysis, we minimize the contribution of this intrinsic non-enzymatic behavior on our $\mathrm{K}_{M}$ calculations. The calculation of $k_{\max }$ follows an irreversible first order decay process and is insulated from that concentration effect.

G. Inhibitor $\mathbf{I C}_{\mathbf{5 0}}$ determination: Inhibitory activity of hit compounds 1-4 and their derivatives 512 toward $\mathrm{C}-\mathrm{H}-\mathrm{Y}$ cholesterolysis were determined from plots of relative initial velocity vs. inhibitor concentration with cholesterol at its $K_{M}$ value of $1.5 \mu \mathrm{M} .{ }^{5}$ Initial velocity values were calculated as the slope of the linear phase of the FRET readings at each inhibitor concentration. Relative velocity (RV) was calculated by dividing the initial velocity with inhibitor by the initial velocity in the absence of inhibitor. $I C_{50}$ values were determined in GraphPad using the following equation:

$$
\text { RV = Bottom + (Top-Bottom }) /\left(1+\left([1] / I C_{50}\right)\right)
$$

Where "bottom" is the minimum initial velocity, "top" is the maximum initial velocity, and [I] is the inhibitor concentration. For inhibitors $4,6, \mathbf{1 0}$, and $\mathbf{1 1}$, top and bottom values were set to 1 and 0.01 respectively. 
H. Inhibitor binding affinity determined by $\triangle F R E T$ assay: The dose-dependent FRET enhancement from $\mathrm{C}-\mathrm{H}-\mathrm{Y}$ and selected $\mathrm{C}-\mathrm{H}-\mathrm{Y}$ mutants accompanying addition of $\mathbf{8}$ (tBT-HBT) was used to calculate inhibitor $K_{D}$ values. Binding assays contained the FRET reporter proteins at $1 \times 10^{-7} \mathrm{M}$ in cholesterolysis buffer but without cholesterol. Average enhancement in FRET relative to no-compound control wells was collected for each condition $(n=3)$ and plotted versus inhibitor concentration. A modified quadratic equation for tight inhibitor binding (below) was used to calculate the apparent binding constant. $\triangle F R E T_{\text {Max }}$ represents the maximum change in FRET from addition of saturating inhibitor. Error in $K_{D}$ determination was reduced by use of the Solver function in Excel.

$\Delta$ FRET $_{\text {obs }}=\Delta$ FRET $_{\text {Max }} * \frac{\left([\mathrm{C}-\mathrm{H}-\mathrm{Y}]+[\mathrm{l}]+\left[\mathrm{K}_{\mathrm{D}}\right]\right)-\sqrt{\left([\mathrm{C}-\mathrm{H}-\mathrm{Y}]+[1]+\left[\mathrm{K}_{\mathrm{D}}\right]\right)-4 *[\mathrm{C}-\mathrm{H}-\mathrm{Y}][1]}}{2[\mathrm{E}]}$

I. Photoaffinity labeling of product HhC by $\mathbf{t S P}$ : Product HhC, lacking an N-terminal peptide, was used for photolabeling. To obtain product $\mathrm{HhC}$, we first expressed and purified the precursor protein, His6-HhC, as previously described. ${ }^{3}$ Removal of the $\mathrm{N}$-terminal His 6 tag from this precursor was carried out by cleavage of the internal thioester at the His 6 / HhC junction with $200 \mathrm{mM}$ beta-mercaptoethanol (BME). This thiolysis reaction was carried out at $16{ }^{\circ} \mathrm{C}$ for 24 hours. BME and unreacted precursor were removed from the product $\mathrm{HhC}$ by dialysis into 500 $\mathrm{ml}$ of $1 \mathrm{x}$ PBS, carried out twice, followed by passage of the dialyzed solution over a Qiagen NiNTA column equilibrated with $10 \mathrm{mM}$ imidazole. Product $\mathrm{HhC}$ was collected in a $75 \mathrm{mM}$ imidazole wash and used for subsequent labeling experiments.

Photoaffinity labeling of purified, product HhC with $t S P$ was carried out at room temperature using a mercury UV lamp located eight $\mathrm{cm}$ from the sample cuvette. The photoaffinity labeling reaction contained product $\mathrm{HhC}(5 \mu \mathrm{M})$ in bis-tris buffer $(0.02 \mathrm{M}, \mathrm{pH} 7.1)$ with $\mathrm{NaCl}(0.1 \mathrm{M}), \mathrm{n}$ dodecylphosphocholine (Fos-12, $0.0015 \mathrm{M}$ ) and L-ascorbic acid (0.005 M). The trans-sterol probe (tSP) was added to a final concentration of $10 \mu \mathrm{M}$. In selected samples, inhibitor 1 was added to a final concentration of $200 \mu \mathrm{M}$. Other control reactions contained cholesterol, added to $200 \mu \mathrm{M}$. After 5 minutes of irradiation, samples were denatured by addition of $18 \mathrm{mg}$ urea (6 M, final).

For click labeling HhC-tSP adduct through the alkyne group on $t S P$, a premixed copper sulfate and tris(benzyltriazolylmethyl)amine (THPTA) solution was added to reaction solution to $1 \mathrm{mM}$ copper sulfate and $5 \mathrm{mM}$ THPTA. OG 488 azide fluorophore, aminoguanidine $\mathrm{HCl}$, and L-ascorbic acid were then added to $50 \mu \mathrm{M}, 5 \mathrm{mM}$ and $5 \mathrm{mM}$ respectively. Click reactions were incubated at $30{ }^{\circ} \mathrm{C}$ for 12 hours. For SDS-PAGE analysis, $20 \mu$ aliquots were removed, quenched with load dye containing DTT and boiled. A Bio-Rad Gel Doc EZ system was used for gel imaging with UV and following Coomassie staining.

To identify residues on $\mathrm{HhC}$ photolabeled by $t S P$, aliquots from the photolabeling reaction were first separated over a C4 Jupiter column (Phenomenex). The following RP-HPLC buffers were used: Solvent A - Water + 0.1\% TFA, Solvent B - Acetonitrile + 0.1\% TFA. Gradient conditions: $25 \%$ B increasing to $55 \%$ B over 20 minutes, with a flow rate of $0.8 \mathrm{ml} / \mathrm{min}$. Protein containing 
fractions were combined, evaporated to dryness, and resuspended in $20 \mu \mathrm{l}$ of deionized water. To validate the HhC-tSP adduct, fractions were copper click labeled with OG 488 azide, as described above. A total of $10 \mu \mathrm{g}$ of the HhC-tSP adduct was then submitted to the Proteomics and Metabolomics Core Facility at Weill Cornell Medicine for MS/MS analysis.

J. Generation of D.me HhC homology model: A homology model of Drosophila melanogaster (D.me) HhC was generated from the available D.me Hedgehog/intein (HINT) subdomain structure (PDB ID: 1at0) and the recently published computational model of the human Sonic HhC Sterol Recognition region (SRR). ${ }^{6}$ To generate a computational model of the D.me SRR structure, we first aligned the sequences of human Sonic SRR and the D.me SRR using ClustalOmega. ${ }^{7}$ This alignment along with the Sonic HhC structure were used as inputs for Modeller $9.25^{8,9}$ to create 200 computational predictions of the D.me SRR structure. The modeled D.me SRR structures were ranked using MODELLER's internal scoring function, and the model with the lowest (best) score was chosen for subsequent modeling of a full-length D.me HhC structure.

Full-length D.me HhC combines the published HINT structure and the newly generated D.me SRR structure. The disposition of the two subdomains was based on structural alignment to the Sonic HhC model. The D.me HhC subdomains were ligated together using the Pymol editing tools. Cholesterol was then placed manually into D.me $\mathrm{HhC}$ with a similar binding orientation to the human Sonic HhC-cholesterol complex. The resulting D.me HhC-cholesterol model was submitted to the CHARMM-GUI ${ }^{10}$ solution builder input generator, using neutralizing ions, and all other default settings. The output structure from CHARMM-GUI was then minimized using GROMACS, ${ }^{11}$ with 5000 steps of steepest decent with a Lennard-Jones cutoff radius of $12 \AA$, followed by 125000 steps of equilibration with all settings default from the CHARMM-GUI GROMACS output.

Lastly, the refined D.me HhC-cholesterol model and the tBT-HBT inhibitor were prepared for docking by generating pdbqt files for each structure using Autodock4. ${ }^{12} \mathrm{~A}$ grid box encompassing the entire D. me HhC model was included in a config file for Autodock Vina, ${ }^{13}$ choosing an output of 9 docked conformations with all other settings default. All outputs were inspected manually, and the lowest energy docked structure was chosen for the ternary complex model, D.me HhCcholesterol- $t$ BT-HBT.

\section{K. Synthesis of inhibitor analogs:}

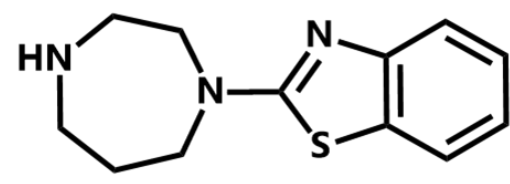

HBT

The homopiperazine-benzothiazole (HBT) fragment was synthesized from homopiperazine and 2-chlorobenzothiazole as previously described. ${ }^{14}$ 
(a) Compound 5: $25.1 \mathrm{mg}$ (.15 mmol, 1.5 eq.) of 1-tert-butyl-1H-pyrrole-3-carboxylic acid, $2.44 \mathrm{mg}(0.02 \mathrm{mmol}, 0.2 \mathrm{eq}$.) of 4-(dimethylamino)pyridine (DMAP), and $38.34 \mathrm{mg}(0.2$ mmol, 2 eq.) of $\mathrm{N}$-(3-dimethylaminopropyl)- $\mathrm{N}^{\prime}$-ethylcarbodiimide hydrochloride (EDC) were mixed in $4 \mathrm{ml}$ of dichloromethane for 30 minutes at room temperature while stirring. Next, $23.3 \mathrm{mg}$ ( $0.1 \mathrm{mmol}, 1$ eq.) of HBT was dissolved in dichloromethane and added to the aforementioned mixture and allowed to react for 4 hours. The solution was aqueous extracted, the organic layer was collected and dried with anhydrous sodium sulfate, and the product was separated via silica gel flash chromatography. Mobile phase: isocratic 1:1 dichloromethane:ethyl acetate. The product was characterized by NMR (pages S16 and S17) and LCMS (page S32). Isolated yield: 90\%.

(b) Compound 6: $11.1 \mathrm{mg}$ (0.1 mmol, 1 eq.) of 1H-pyrrole-3-carboxylic acid, was reacted with DMAP, EDC, and HBT as with compound 5, and purified similarly. Reaction solvent: $50 \%$ acetone/50\% dichloromethane (v/v). Column mobile phase: isocratic 9:1 ethyl acetate:ethanol. The product was characterized by NMR (pages S18 and S19) and LCMS (page S32). Isolated yield: 66\%.

(c) Compound 7: $16.9 \mathrm{mg}$ (0.1 mmol, 1 eq.) of 2-tert-butyl-1,3-oxazole-5-carboxylic acid, was reacted with DMAP, EDC, and HBT as with compound 5, and purified similarly. Column mobile phase: isocratic 9:1 ethyl acetate:ethanol. The product was characterized by NMR (pages S20 and S21) and LCMS (page S32). Isolated yield: 68\%.

(d) Compound 8 (tBT-HBT): $18.4 \mathrm{mg}$ ( $0.1 \mathrm{mmol}, 1$ eq.) of 5-tert-butylthiophene-2-carboxylic acid, was reacted with DMAP, EDC, and HBT as with compound 5 , and purified similarly. Column mobile phase: isocratic 9:1 ethyl acetate:ethanol. The product was characterized by NMR (pages S22 and S23) and LCMS (page S32). Isolated yield: 44\%.

(e) Compound 9: $15 \mathrm{mg}$ (0.1 mmol, 1 eq.) of sodium thiophene-2-carboxylate, was reacted with DMAP, EDC, and HBT as with compound 5, and purified similarly. Column mobile phase: isocratic 9:1 dichloromethane:methanol. The product was characterized by NMR (pages S24 and S25) and LCMS (page S32). Isolated yield: $80 \%$.

(f) Compound 10: $20.4 \mathrm{mg}$ (0.1 mmol, 1 eq.) of 5-phenylthiophene-2-carboxylic acid was reacted with DMAP, EDC, and HBT as with compound 5, and purified similarly. Column mobile phase: isocratic 5:5 ethyl acetate:dichloromethane. The product was characterized by NMR (pages S26 and S27) and LCMS (page S32). Isolated yield: $90 \%$.

(g) Compound 11: $15.3 \mathrm{mg}$ (0.1 mmol, 1 eq.) of 5-cyanothiophene-2-carboxylic acid, was reacted with DMAP, EDC, and $\mathrm{HBT}$ as with compound 5, and purified similarly. Column mobile phase: isocratic 9:1 dichloromethane:methanol. The product was characterized by NMR (pages S28 and S29) and LCMS (page S32). Isolated yield: 76\%. 
(h) Compound 12: $20.7 \mathrm{mg}$ (0.1 mmol, 1 eq.) 5-bromo-2-thiophenecarboxylic acid, was reacted with DMAP, EDC, and HBT as with compound 5, and purified similarly. Column mobile phase: isocratic 9:1 dichloromethane:methanol. The product was characterized by NMR (pages S30 and S31) and LCMS (page S32). Isolated yield: 82\%. 


\section{L. ${ }^{1} \mathrm{H},{ }^{13} \mathrm{C}$ NMR spectra for compounds 5-12}
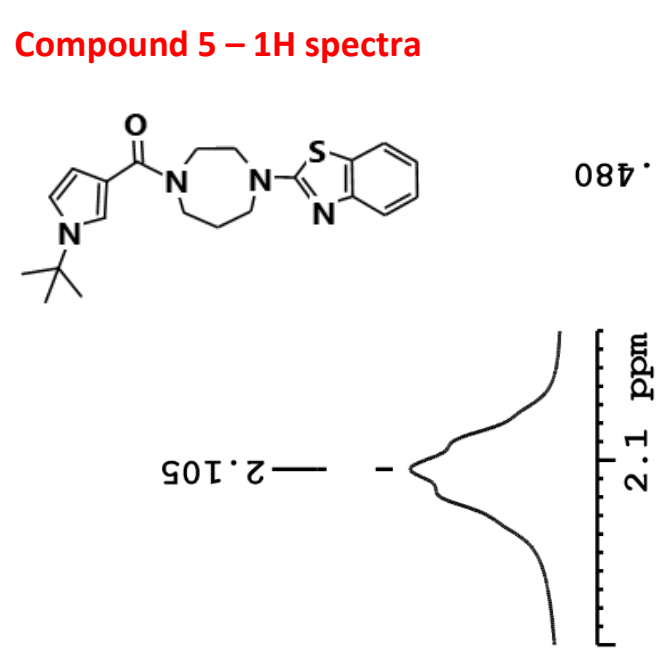

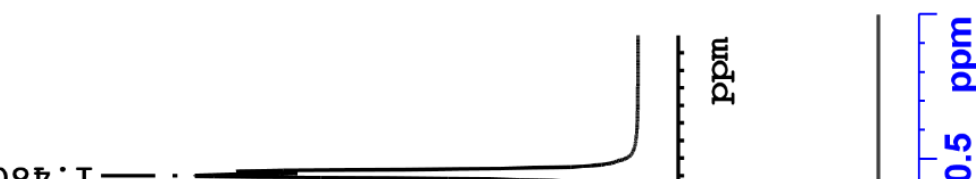

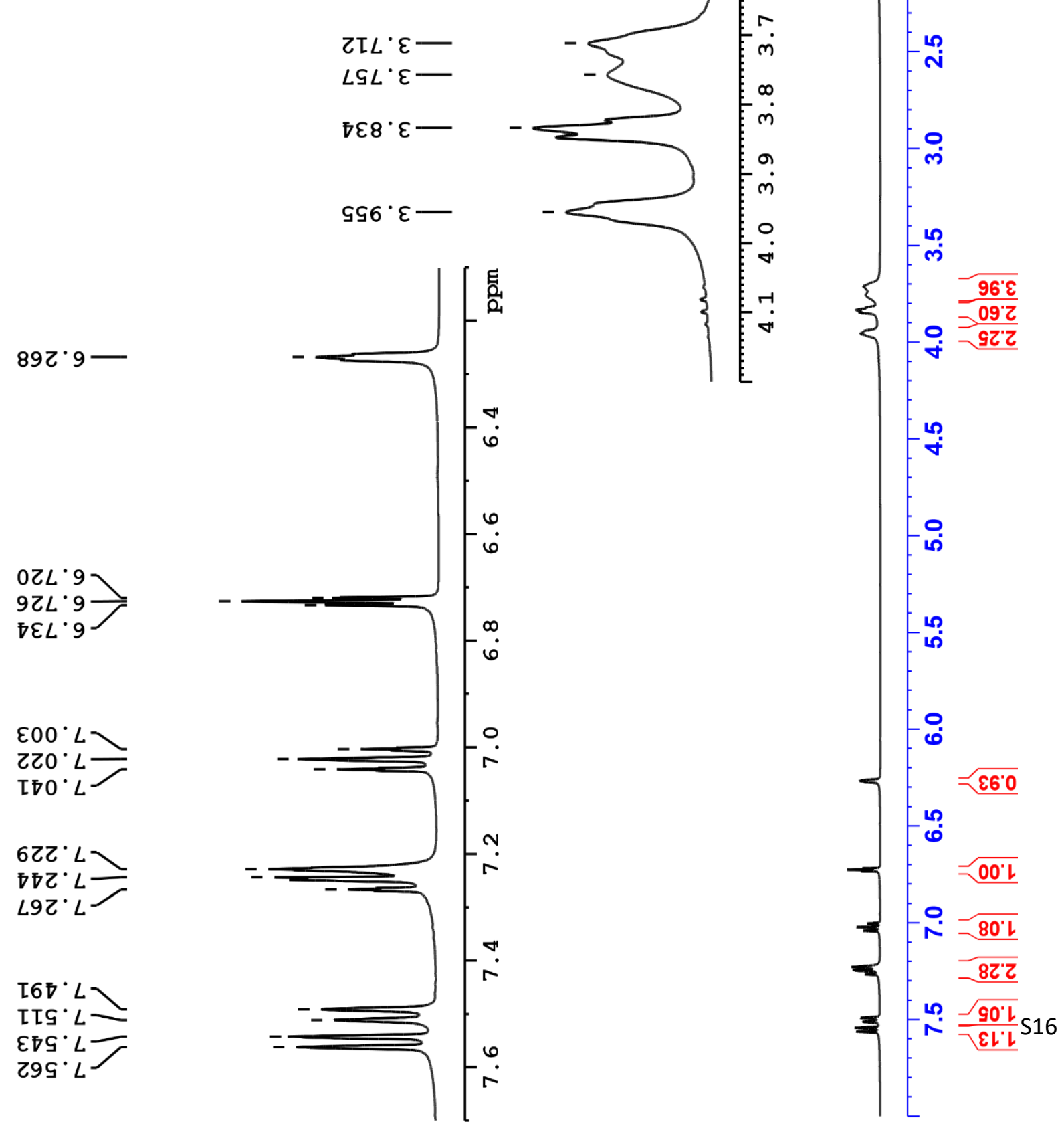


Compound 5 - 13C Spectra

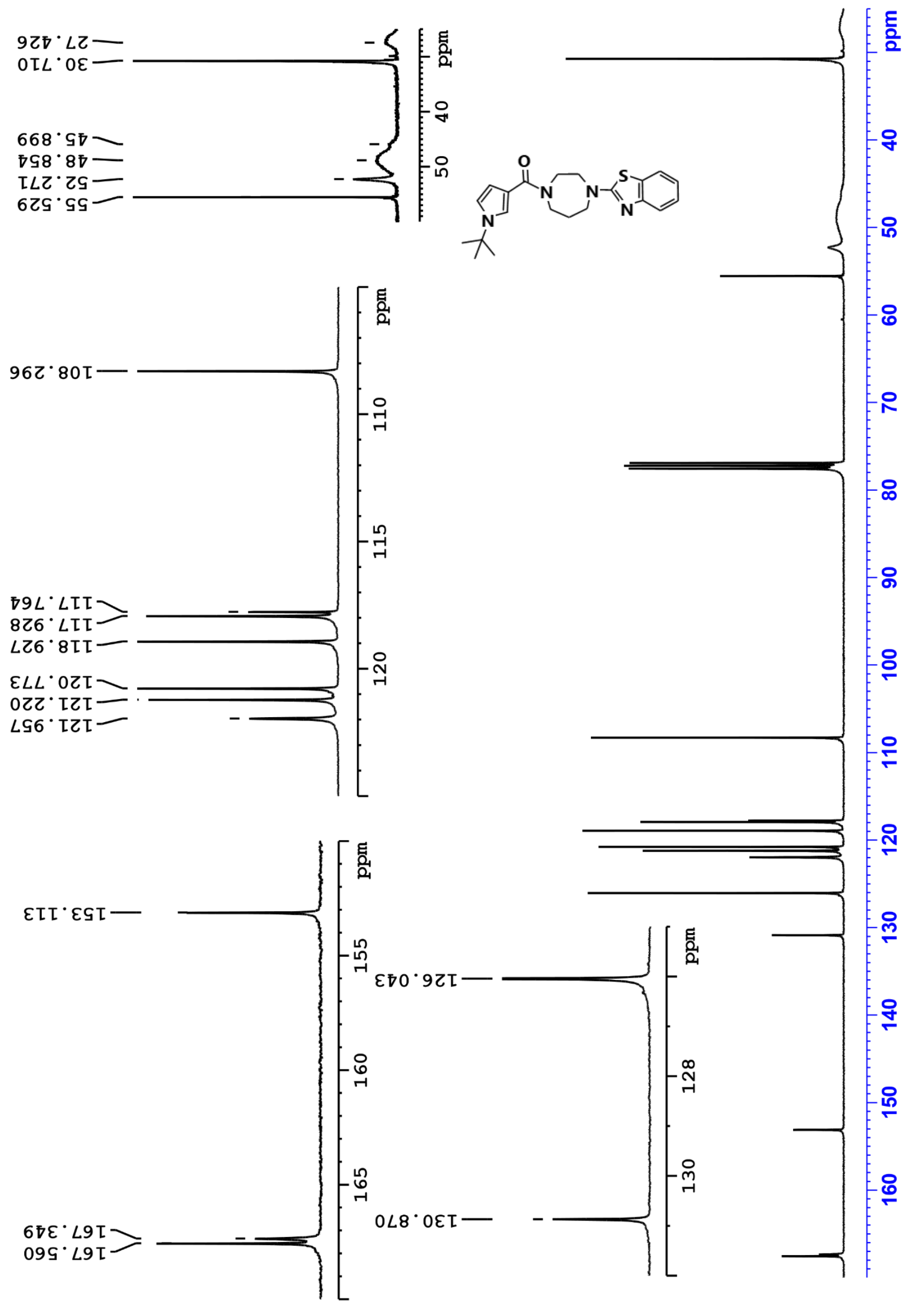




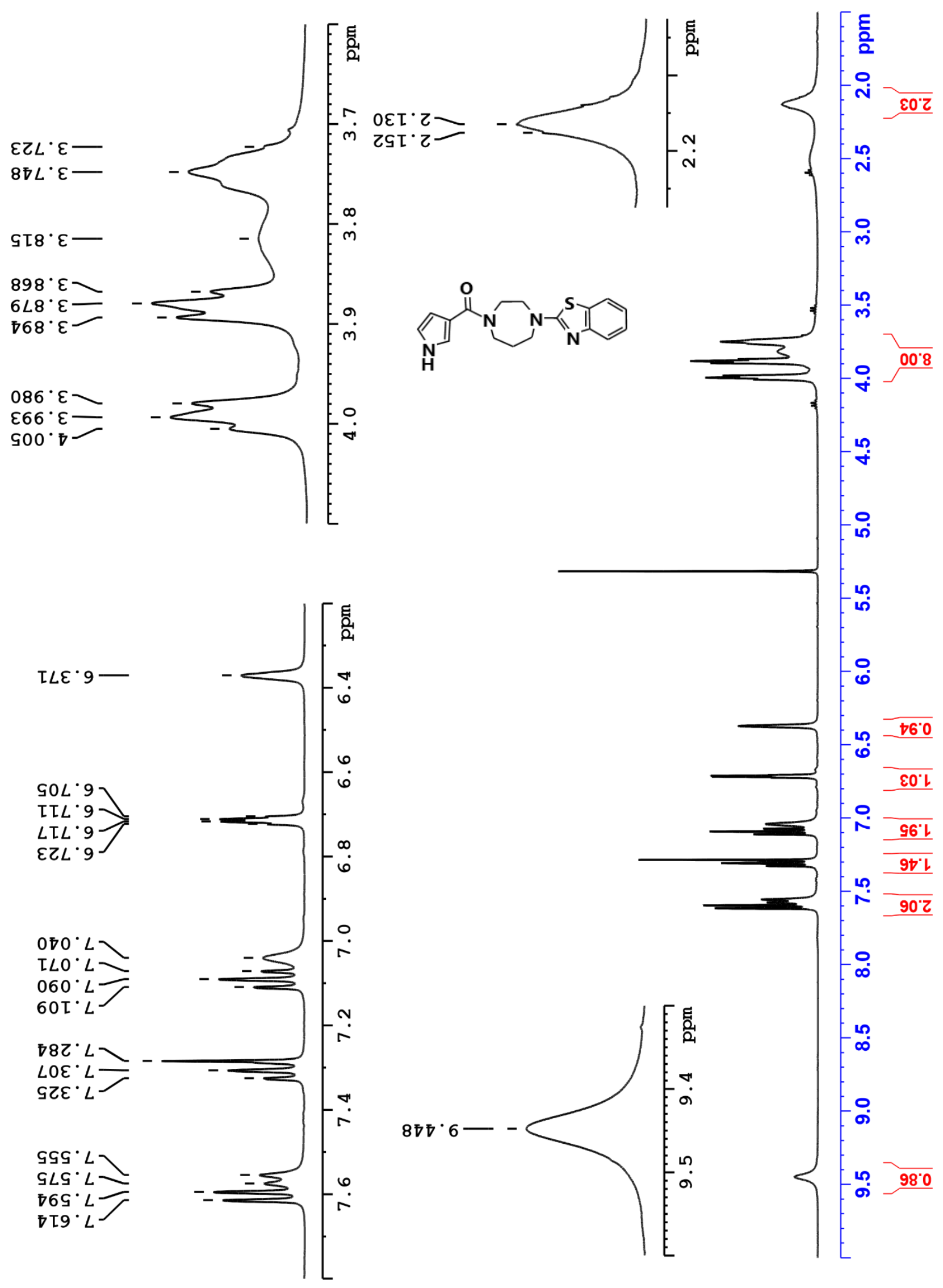




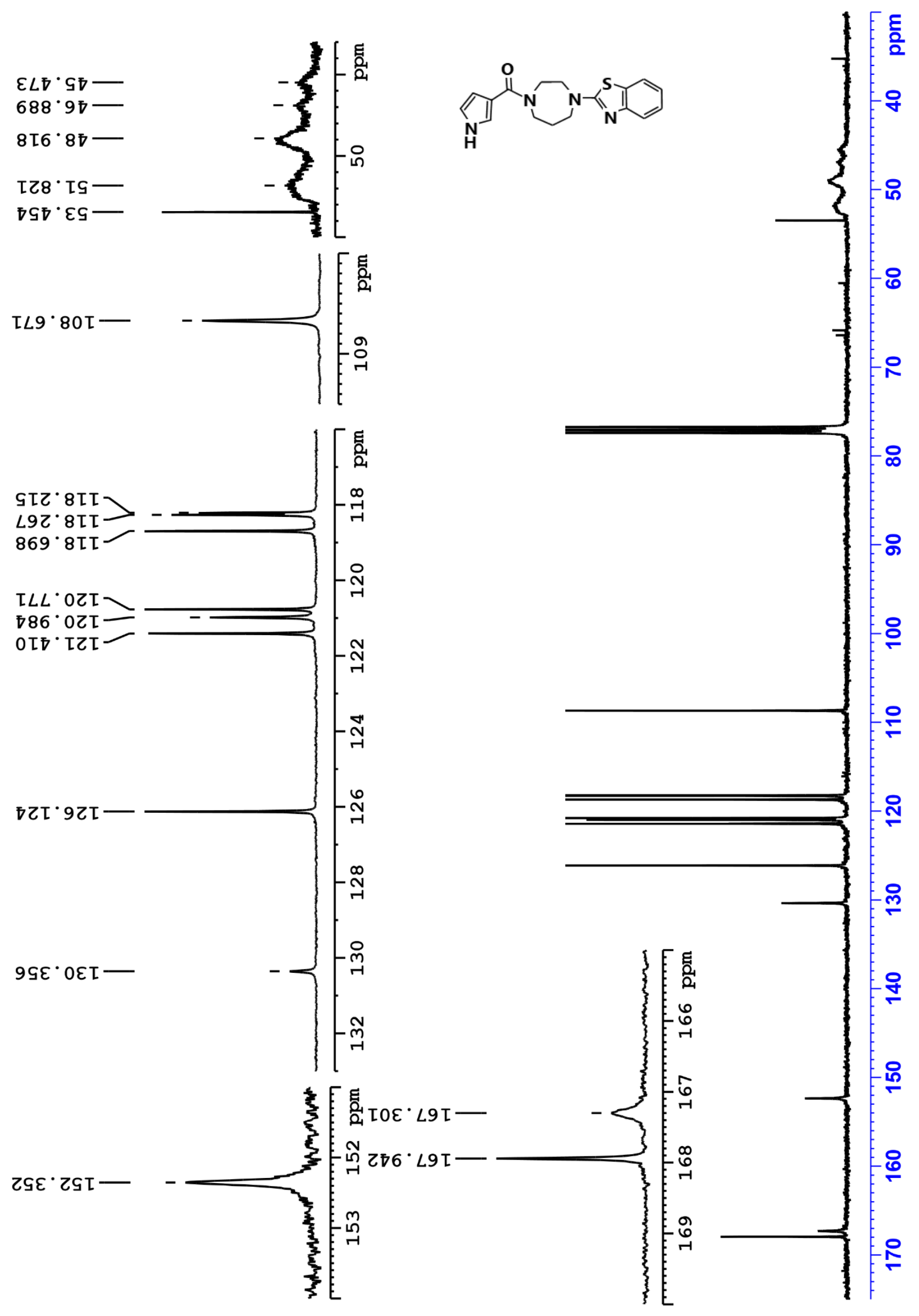


Compound 7 - 1H Spectra

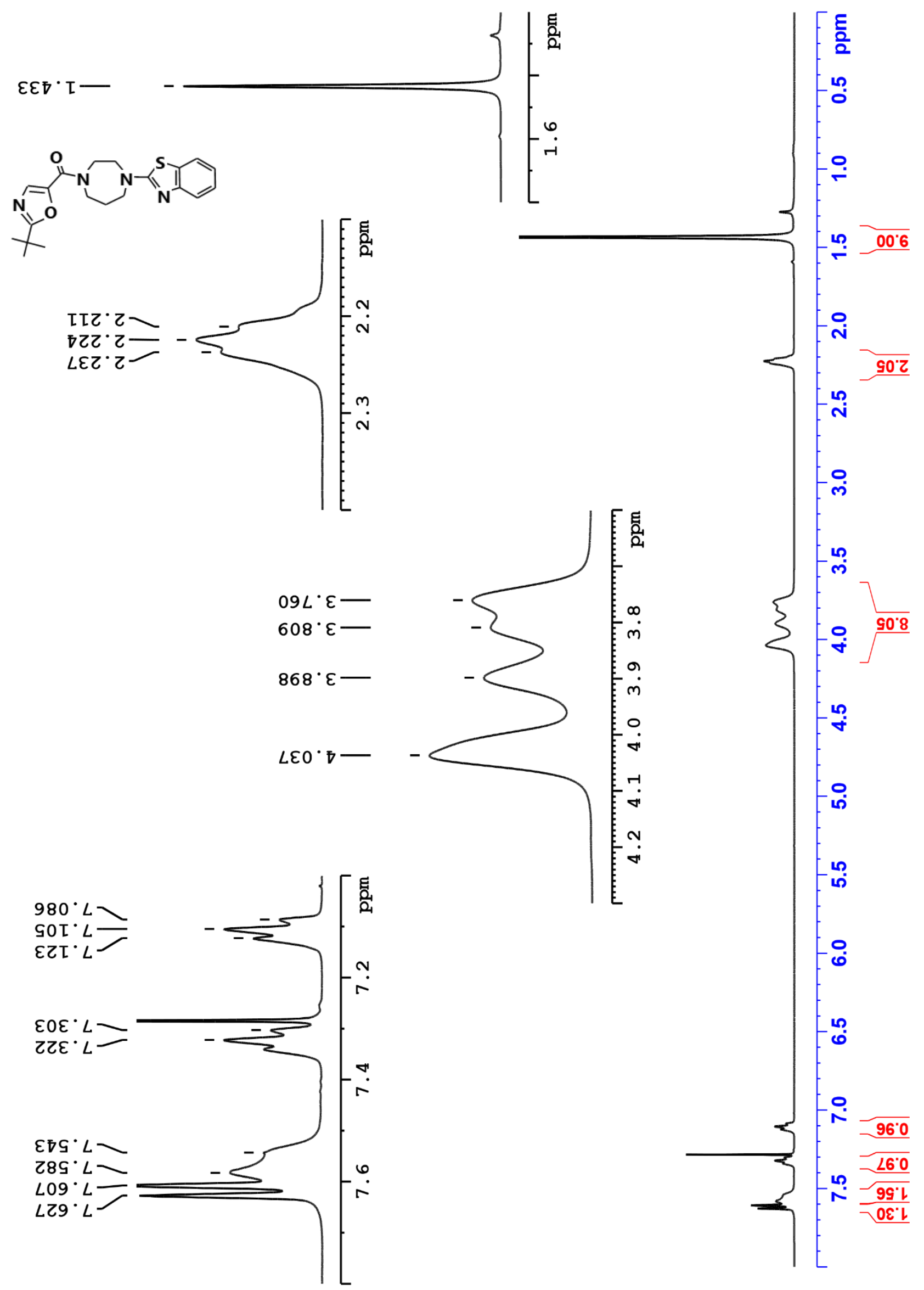




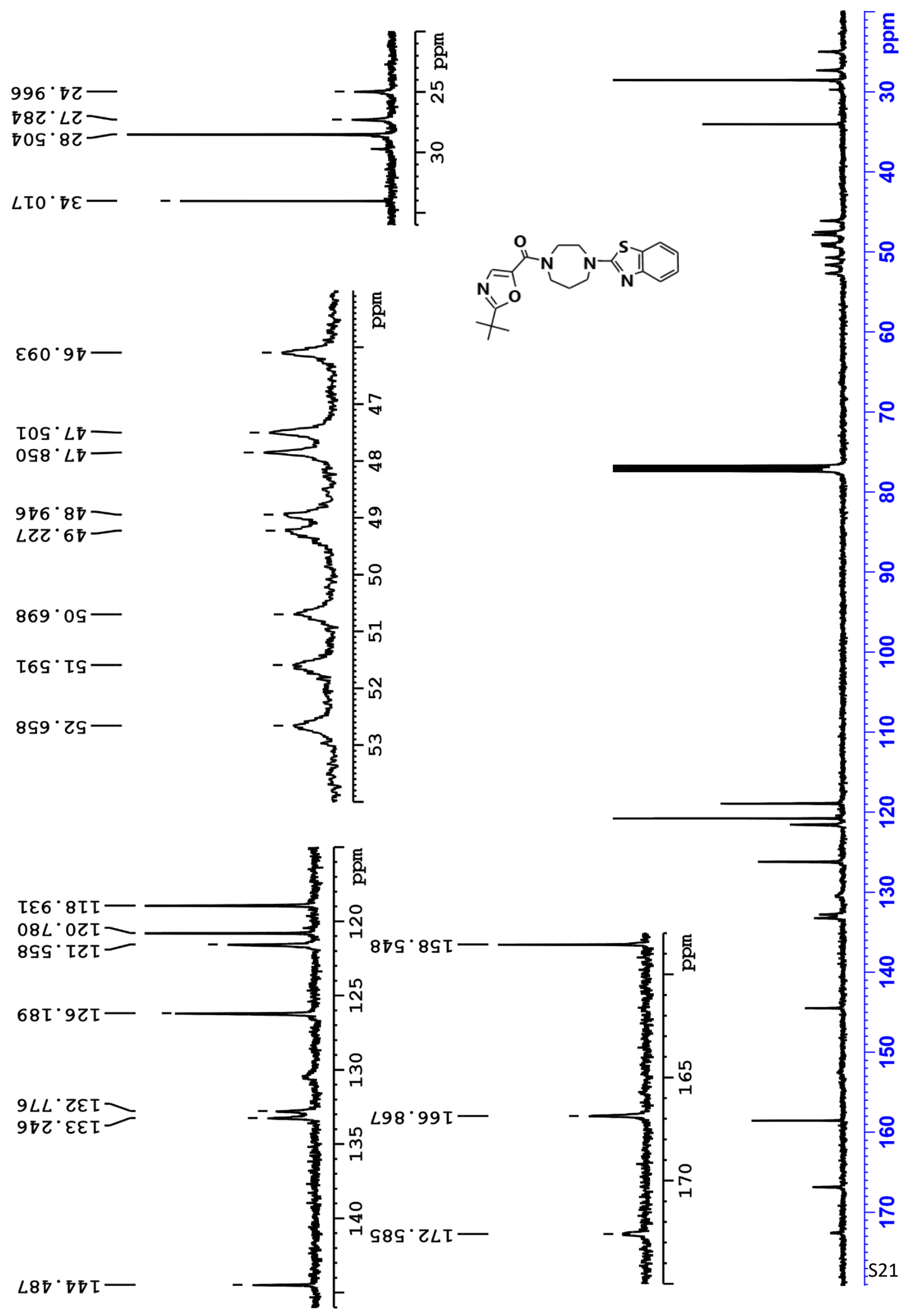




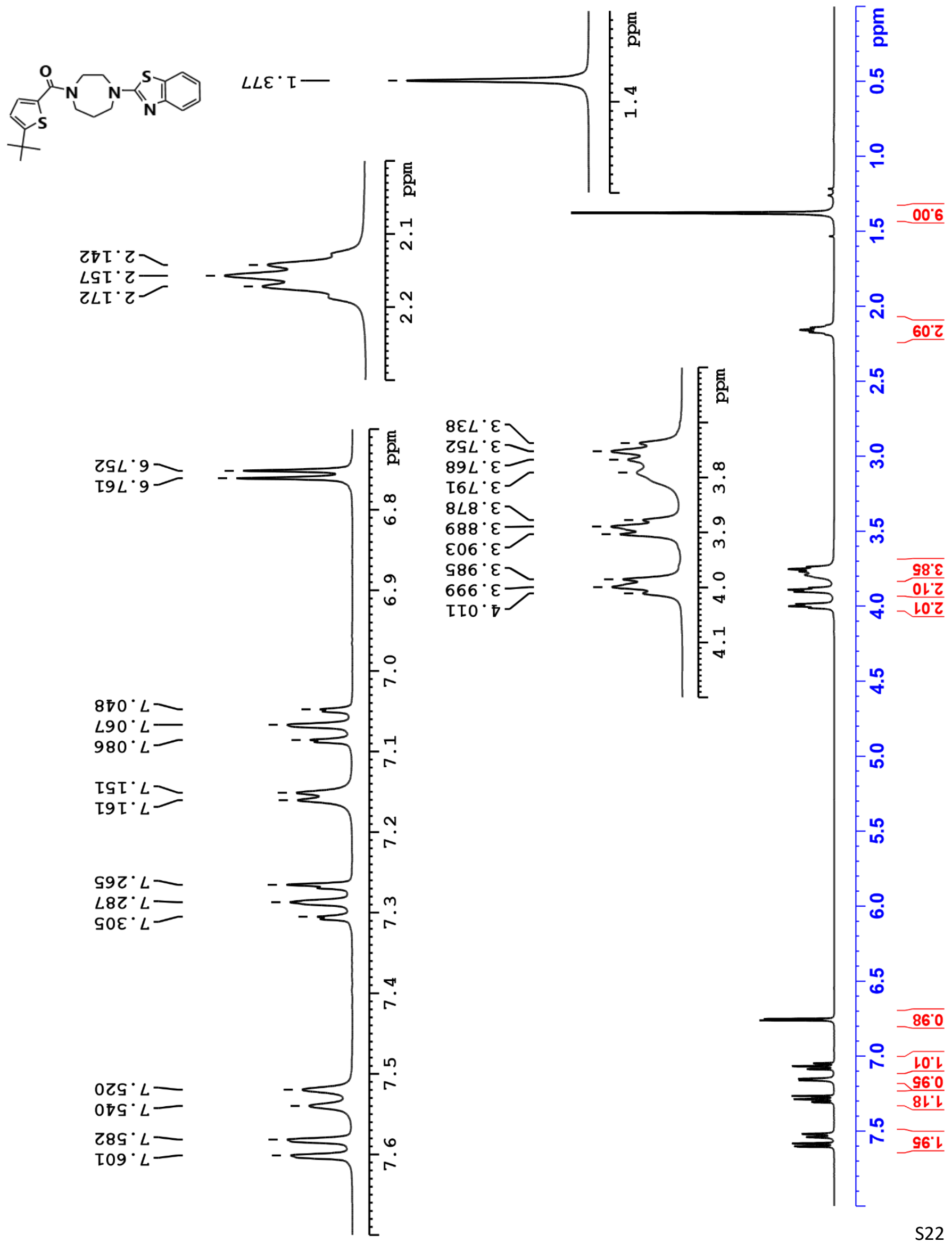




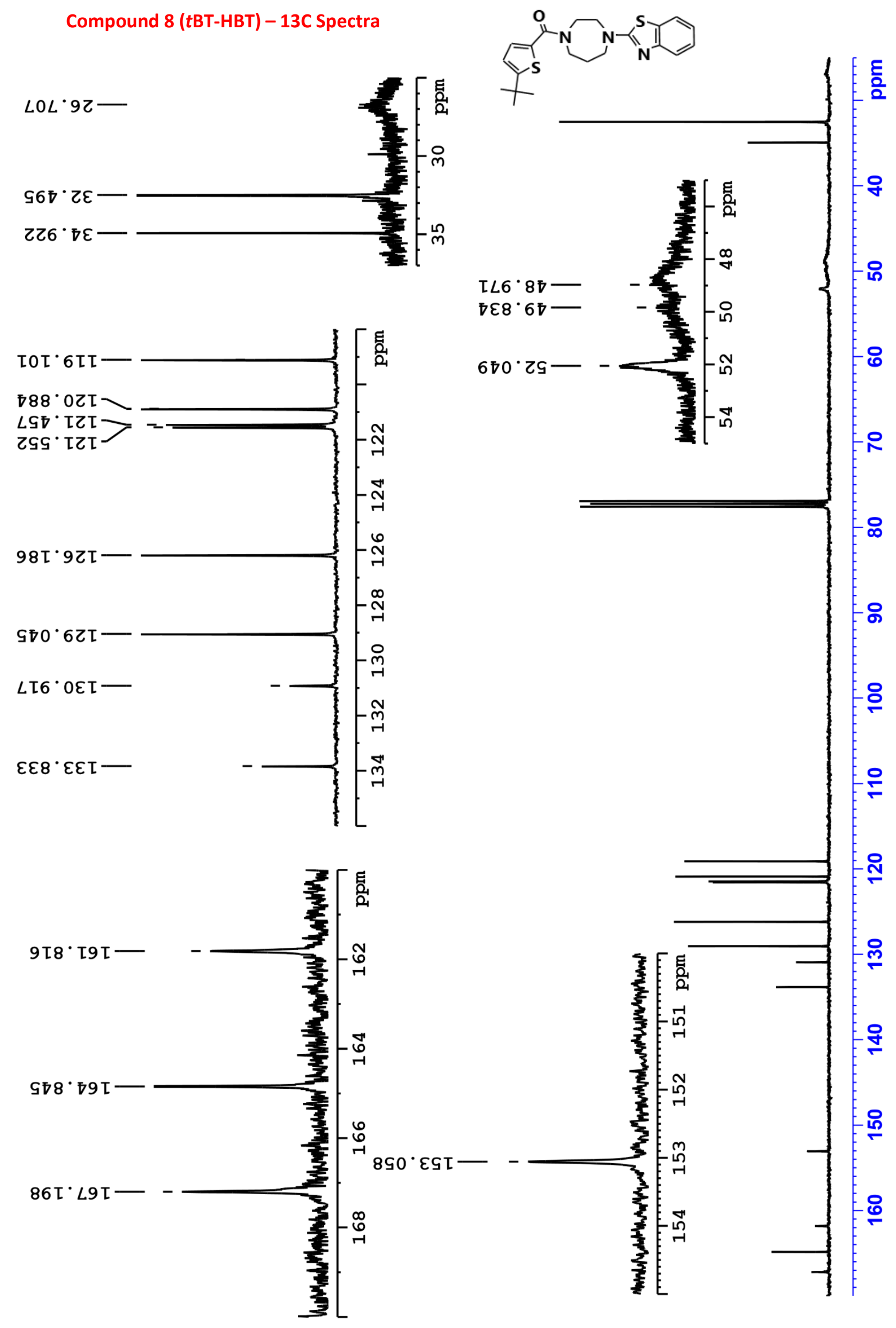


Compound 9-1H Spectra

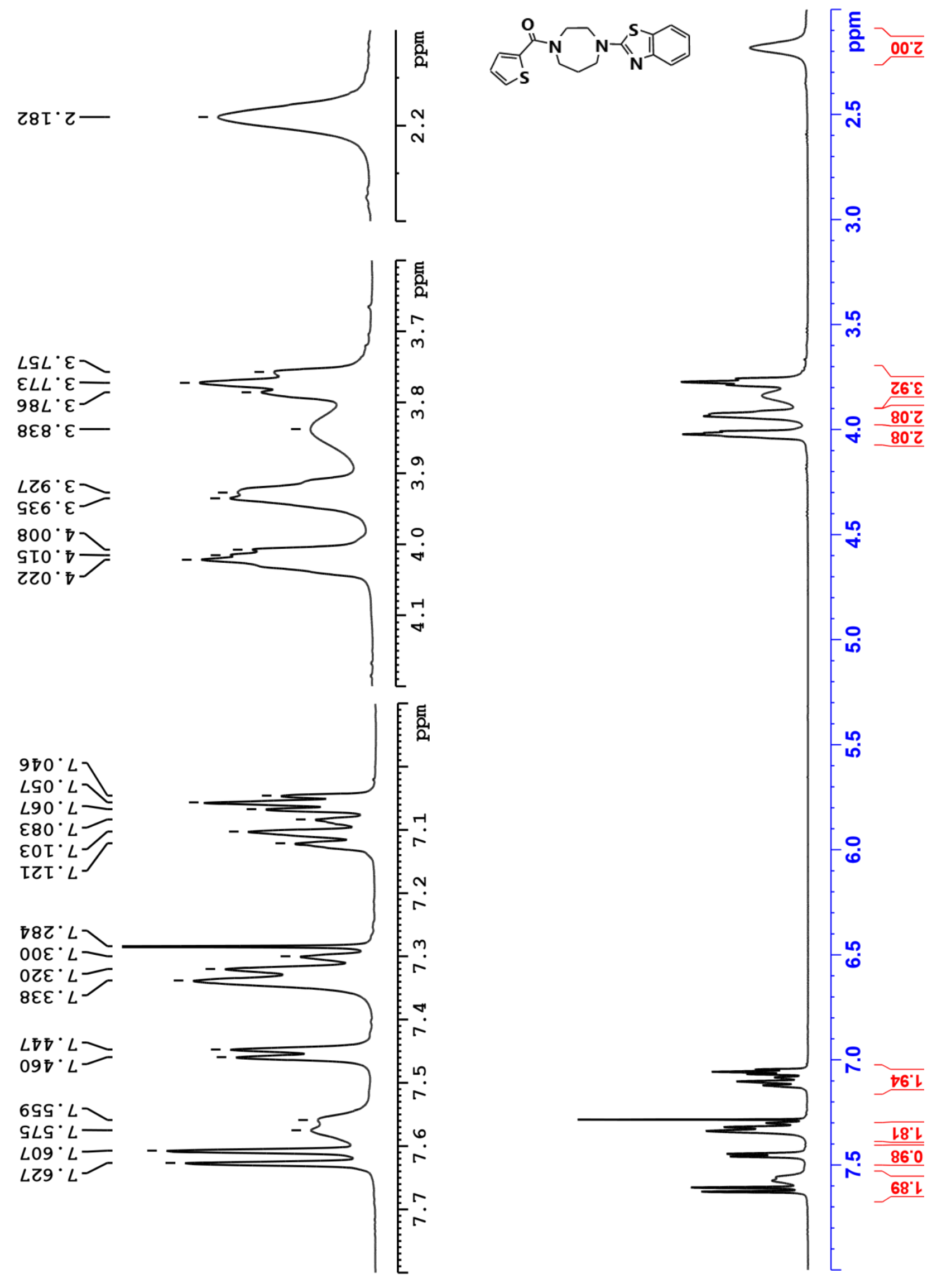




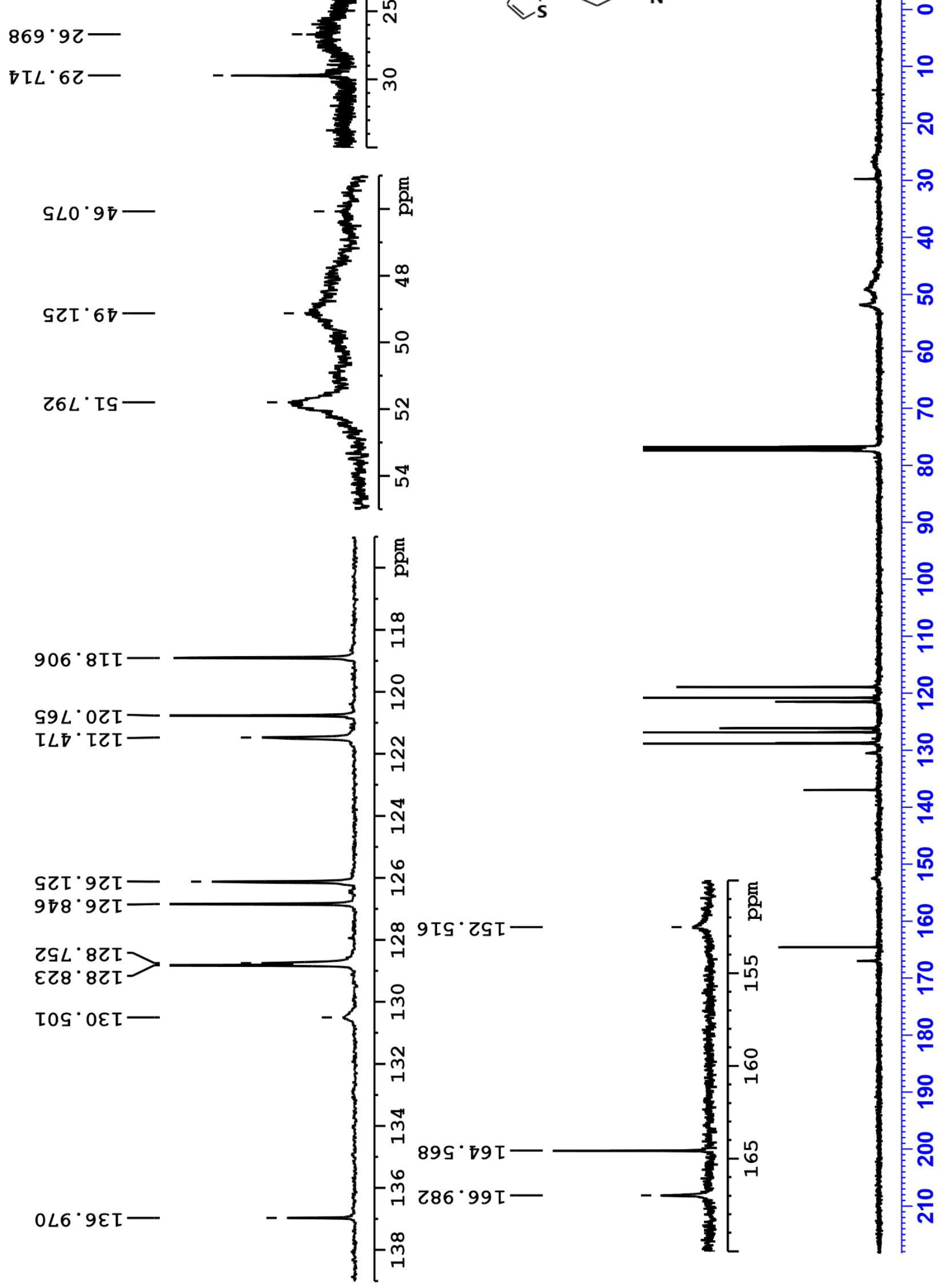


760'

$869^{\circ} \varepsilon>$
$80 L^{\circ} \varepsilon$

ธะ8 $8^{\circ} \varepsilon>$
$\nabla 8^{\circ} \varepsilon$

$\left\llcorner ฤ 6^{\circ} \varepsilon-\right.$
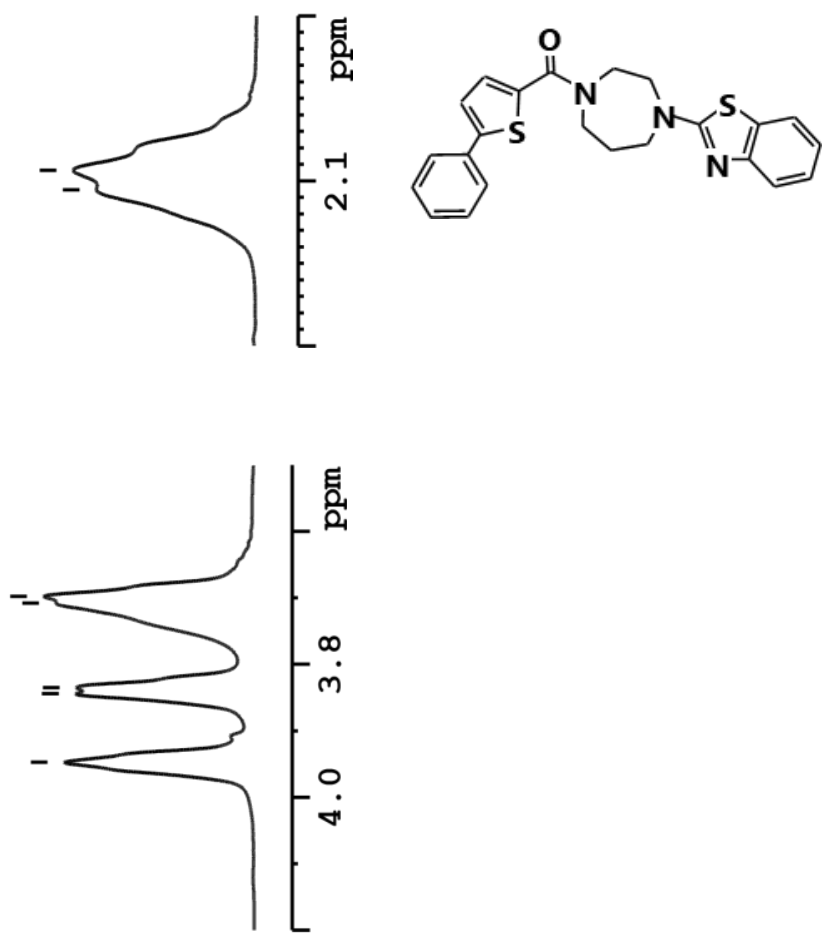

ZL6:9-
L66
OLO

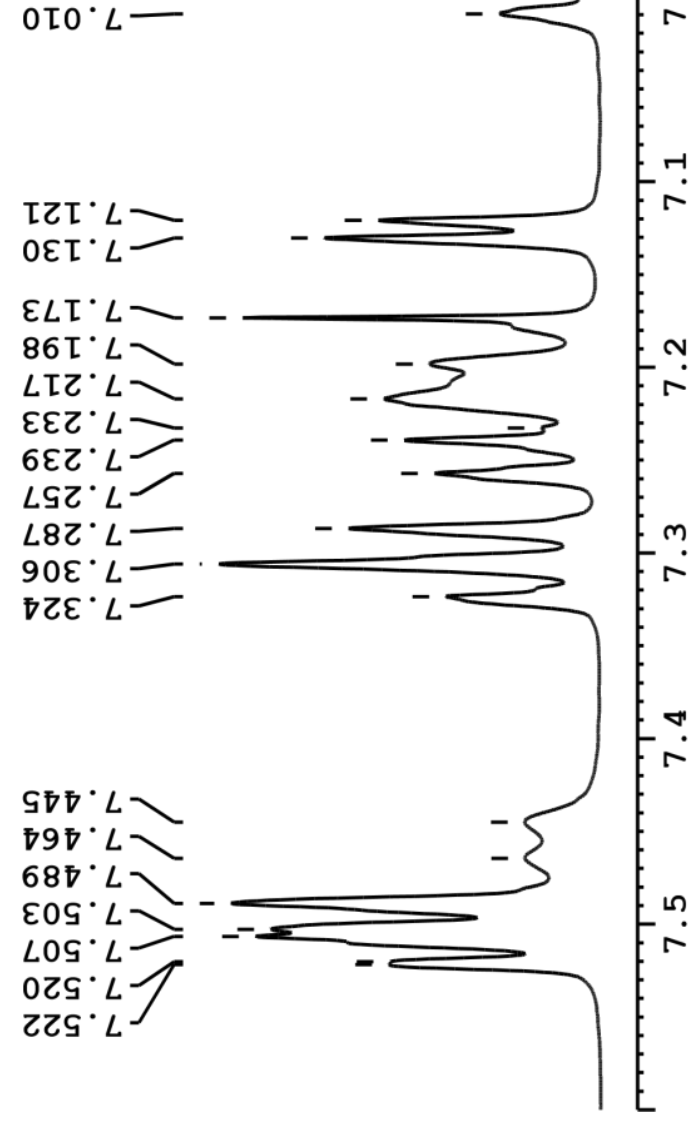

흔 $\longdiv { 0 0 ' z }$

ก

N

m.

m

ल)

m

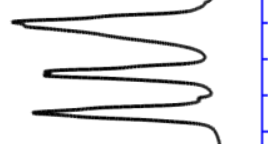

$\left[\begin{array}{ll} & \nabla L 8 \\ -8 & \end{array}\right.$

8

텽

$+$

웅

10

10

10

0

0.

-

")

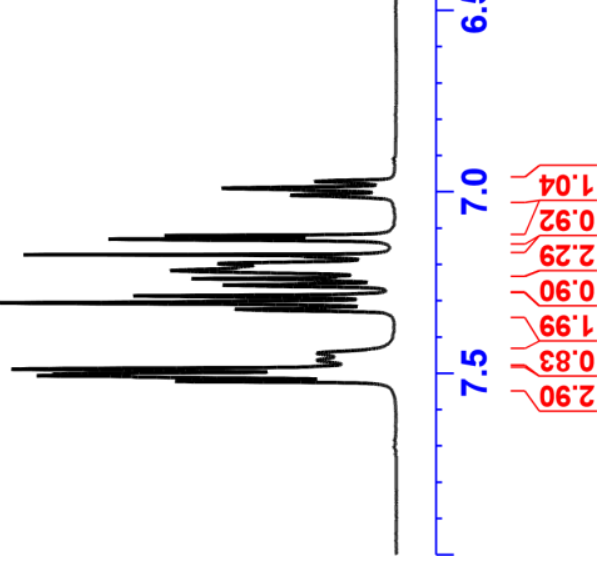




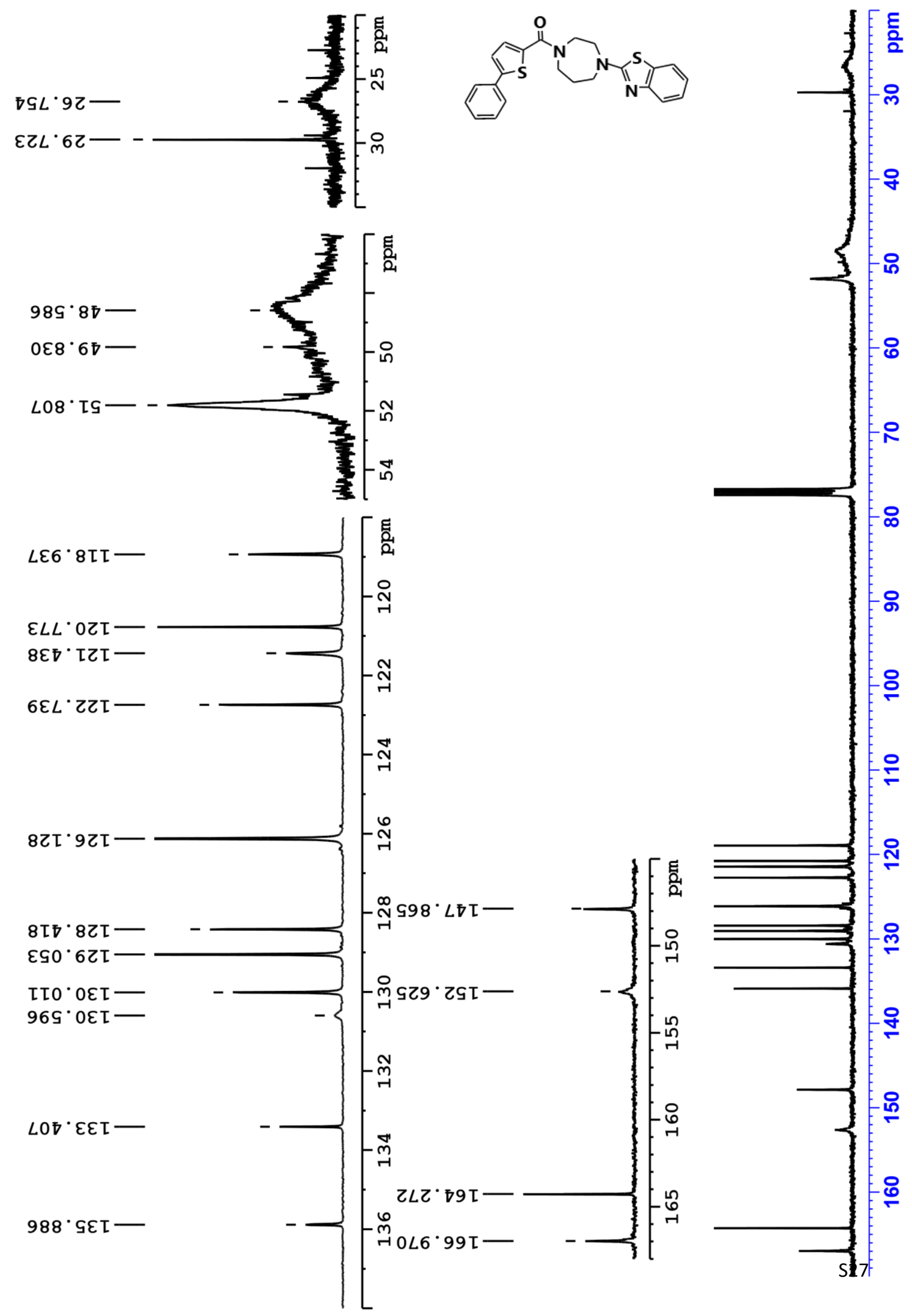




\section{Compound $11-1 \mathrm{H}$ Spectra}

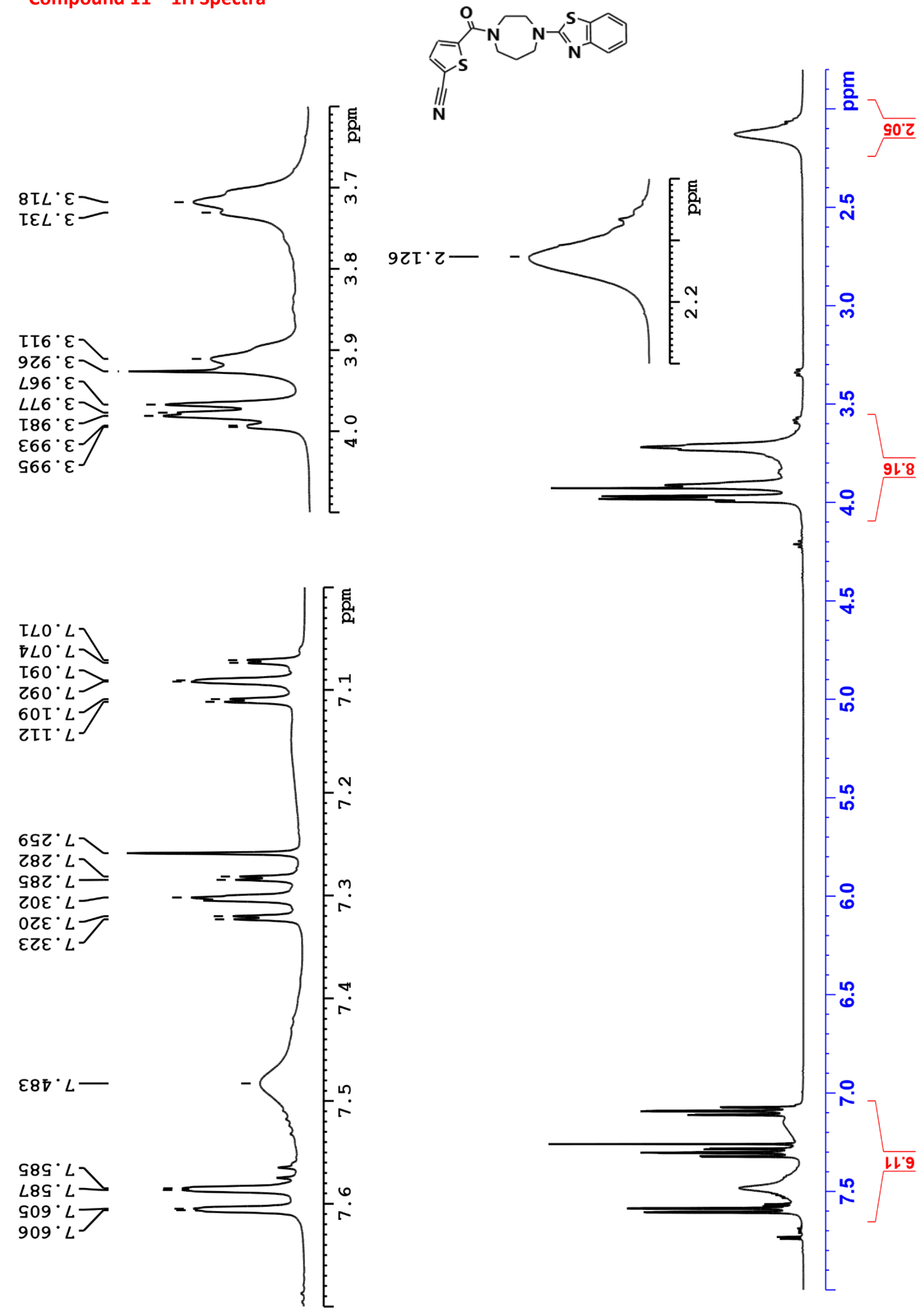


Compound 11 - 13C Spectra

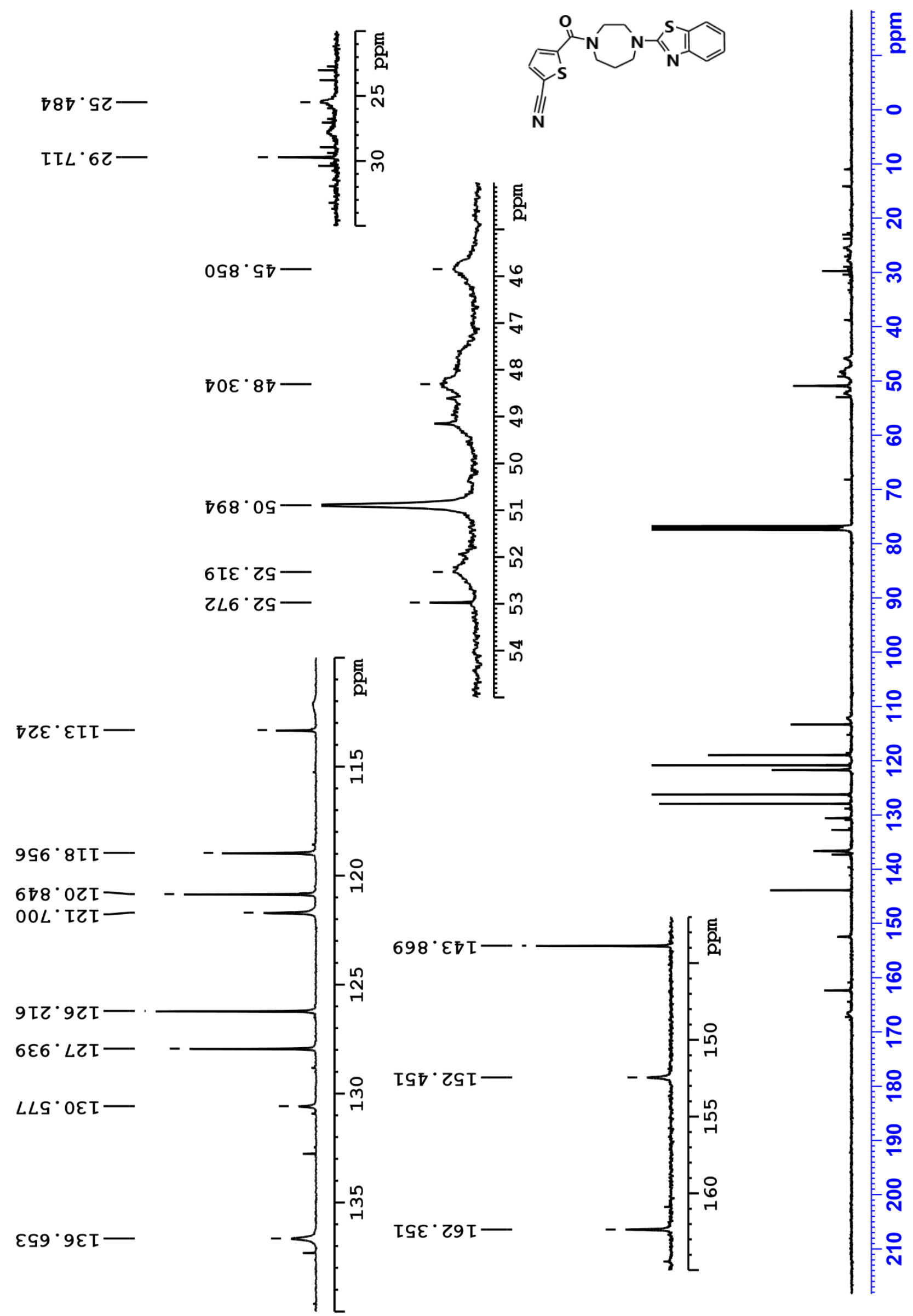


Compound 12-1H Spectra

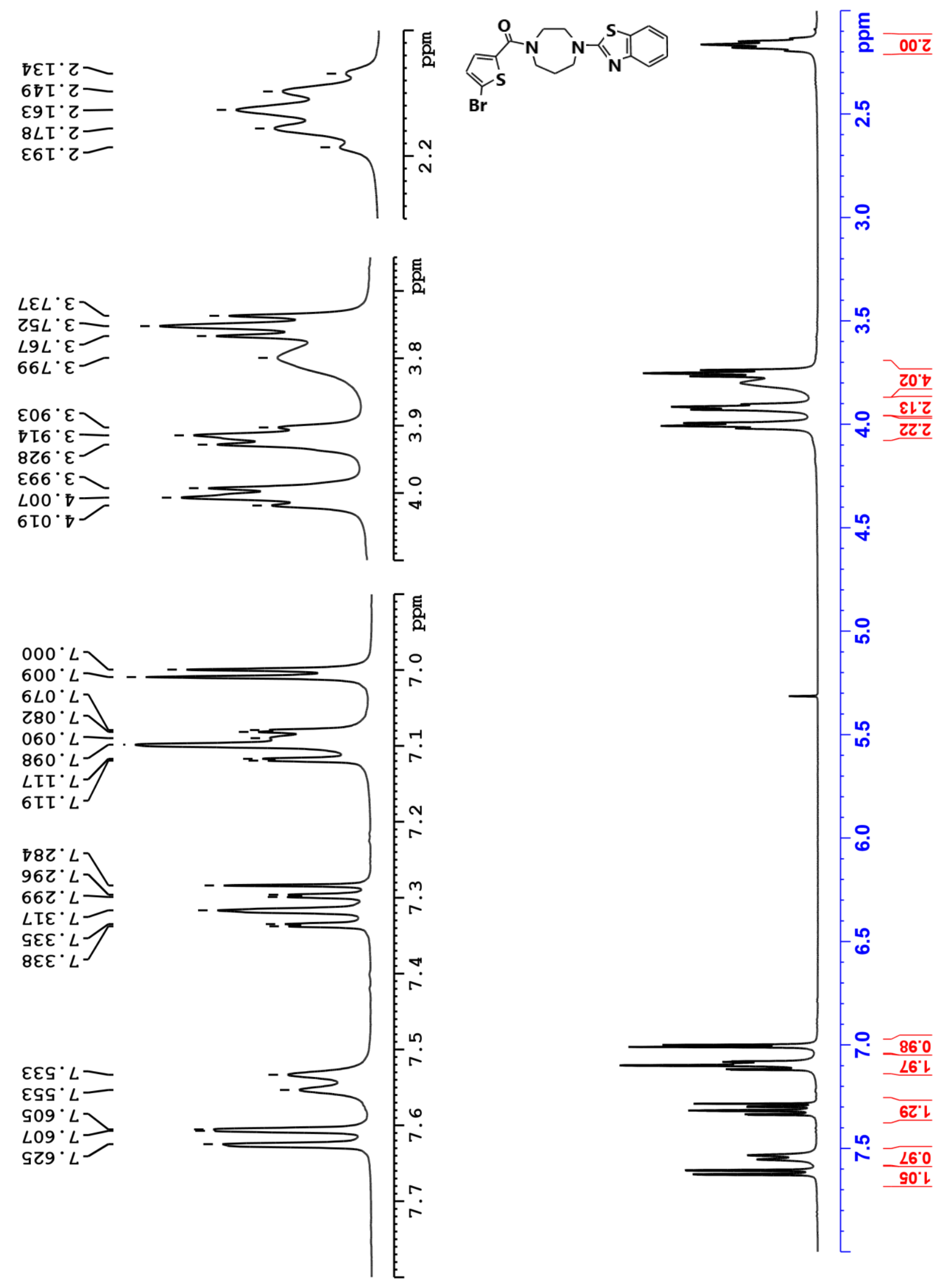



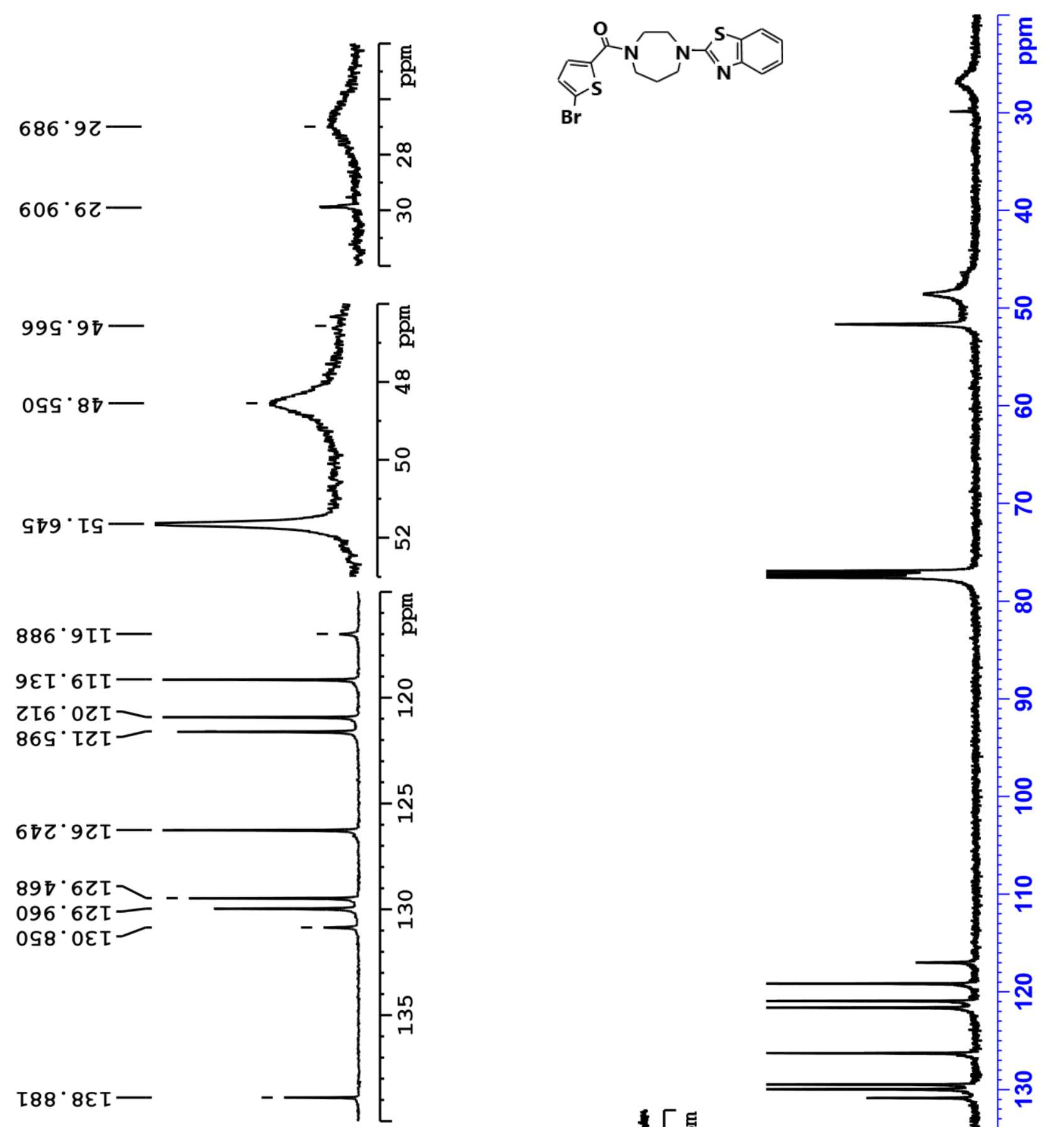

요

8

s

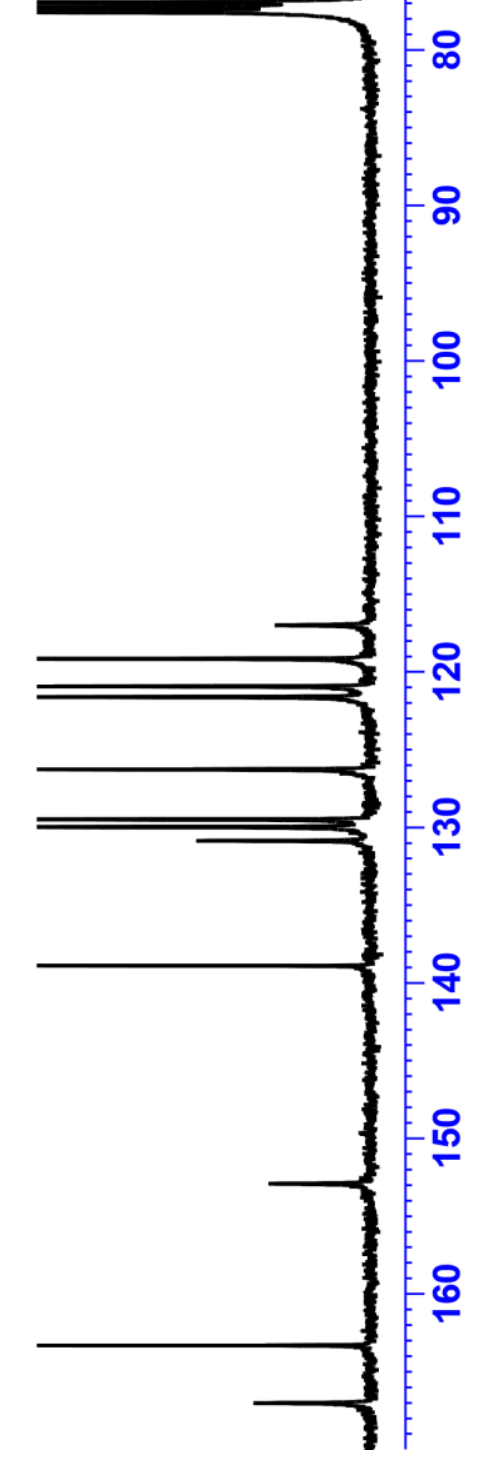




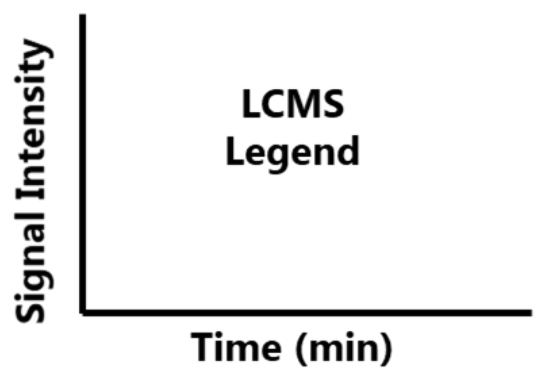

Compound 7

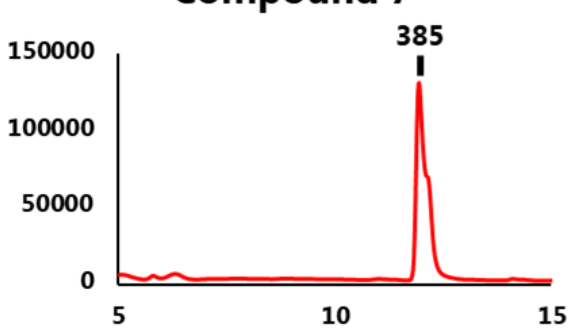

Compound 10

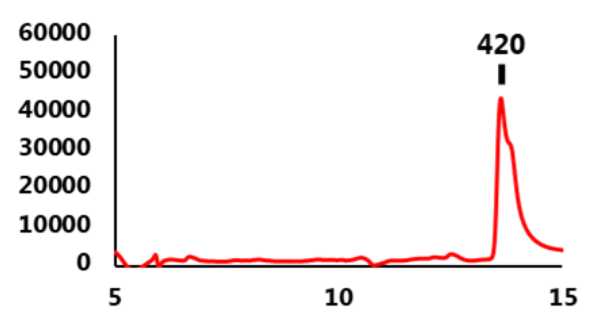

Compound 5

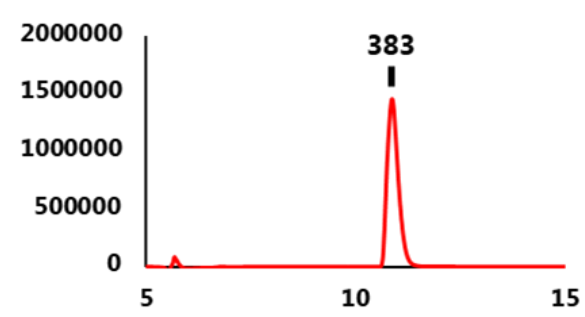

Compound 8

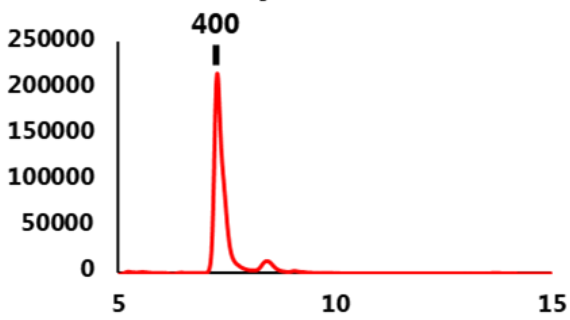

Compound 11

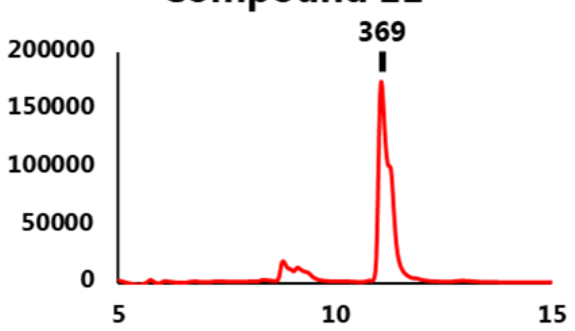

Compound 6

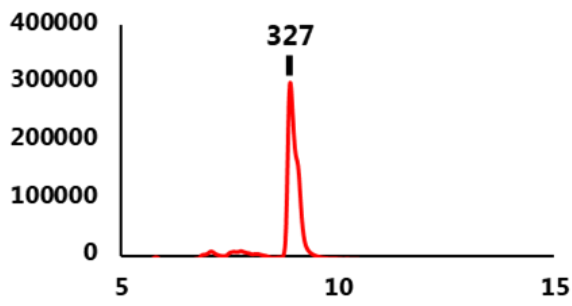

Compound 9

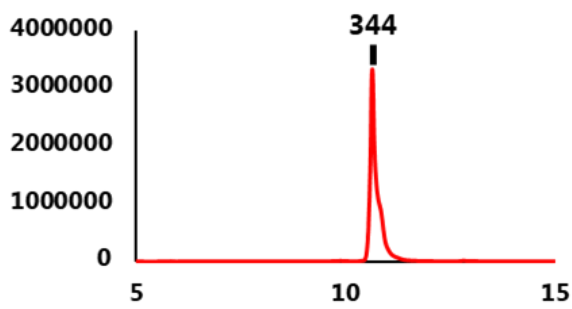

Compound 12

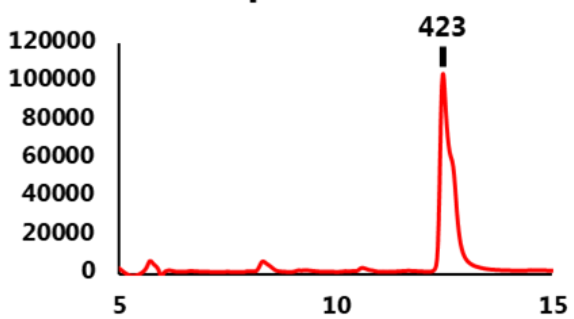

LC traces of synthesized compounds 5-12, with denoted mass of major peaks $(\mathrm{g} / \mathrm{mol})$. 


\section{References}

[1] Owen, T. S., Ngoje, G., Lageman, T. J., Bordeau, B. M., Belfort, M., and Callahan, B. P. (2015) Forster resonance energy transfer-based cholesterolysis assay identifies a novel hedgehog inhibitor, Anal Biochem 488, 1-5.

[2] Owen, T. S., Xie, X. J., Laraway, B., Ngoje, G., Wang, C., and Callahan, B. P. (2015) Active site targeting of hedgehog precursor protein with phenylarsine oxide, Chembiochem 16, 55-58.

[3] Bordeau, B. M., Ciulla, D. A., and Callahan, B. P. (2016) Hedgehog Proteins Consume Steroidal CYP17A1 Antagonists: Potential Therapeutic Significance in Advanced Prostate Cancer, ChemMedChem 11, 1983-1986.

[4] Amitai, G., Callahan, B., Stanger, M. J., Belfort, G., and Belfort, M. (2009) Modulation of intein activity by its neighboring extein substrates, Proc Natl Acad Sci U S A 106, 11005-11010.

[5] Ciulla, D. A., Jorgensen, M. T., Giner, J. L., and Callahan, B. P. (2018) Chemical Bypass of General Base Catalysis in Hedgehog Protein Cholesterolysis Using a Hyper-Nucleophilic Substrate, J Am Chem Soc 140, 916-918.

[6] Mafi, A., Purohit, R., Vielmas, E., Lauinger, A. R., Lam, B., Cheng, Y. S., Zhang, T., Huang, Y., Kim, S. K., Goddard, W. A., 3rd, and Ondrus, A. E. (2021) Hedgehog proteins create a dynamic cholesterol interface, PLoS One 16, e0246814.

[7] Sievers, F., Wilm, A., Dineen, D., Gibson, T. J., Karplus, K., Li, W., Lopez, R., McWilliam, H., Remmert, M., Soding, J., Thompson, J. D., and Higgins, D. G. (2011) Fast, scalable generation of high-quality protein multiple sequence alignments using Clustal Omega, Mol Syst Biol 7, 539.

[8] Sali, A. (1996) Comparative protein modeling by satisfaction of spatial restraints, Acta Crystallographica Section A Foundations of Crystallography 52, C88-C88.

[9] Webb, B., and Sali, A. (2016) Comparative Protein Structure Modeling Using MODELLER, Curr Protoc Bioinformatics 54, 56 1-5 637.

[10] Jo, S., Kim, T., Iyer, V. G., and Im, W. (2008) CHARMM-GUI: a web-based graphical user interface for CHARMM, J Comput Chem 29, 1859-1865.

[11] Van Der Spoel, D., Lindahl, E., Hess, B., Groenhof, G., Mark, A. E., and Berendsen, H. J. (2005) GROMACS: fast, flexible, and free, J Comput Chem 26, 1701-1718.

[12] Morris, G. M., Huey, R., Lindstrom, W., Sanner, M. F., Belew, R. K., Goodsell, D. S., and Olson, A. J. (2009) AutoDock4 and AutoDockTools4: Automated docking with selective receptor flexibility, $J$ Comput Chem 30, 2785-2791.

[13] Trott, O., and Olson, A. J. (2010) AutoDock Vina: improving the speed and accuracy of docking with a new scoring function, efficient optimization, and multithreading, $J$ Comput Chem 31, 455-461.

[14] Smith, C. J., Wagner, A. G., Stagnitta, R. T., Xu, Z., Pezzullo, J. L., Giner, J. L., Xie, J., Covey, D. F., Wang, C., and Callahan, B. P. (2020) Subverting Hedgehog Protein Autoprocessing by Chemical Induction of Paracatalysis, Biochemistry 59, 736-741. 